\title{
Hydrodynamics influence on light conversion in photobioreactors: An energetically consistent analysis
}

\author{
J. Pruvost ${ }^{\mathrm{a}, *}$, J.-F. Cornet ${ }^{\mathrm{b}}$, J. Legrand ${ }^{\mathrm{a}}$ \\ auniversité de Nantes, CNRS, GEPEA UMR-CNRS 6144, Bd de l'Université, CRTT-BP 406, 44602 Saint-Nazaire Cedex, France \\ ${ }^{\mathrm{b}}$ Laboratoire de Génie Chimique et Biochimique, Université Blaise Pascal-Bât. CUST. 24 avenue des Landais, BP 206, F 63174 AUBIERE Cedex, France
}

\begin{abstract}
A B S T R A C T
Hydrodynamics conditions are supposed to affect light conversion in photobioreactors (PBRs), by modifying light availability of suspended photosynthetic cells. The present study aims at rigorously analyzing mixing conditions influence on PBR efficiency. Investigation is based on a Lagrangian formulation, which is well adapted to characterize individual cell history. Its association with a radiative-transfer model to characterize light availability is emphasized.

Such approach has already been applied in PBR modeling, but with an over-simplified formulation where both cell trajectories and radiative transfer were solved independently. As energetic balances on the material and photonic phases will show, it is however necessary to introduce influence of the heterogeneous light access implied by non-ideal mixing conditions in the non-linear radiation field resolution. The proposed energetically consistent Lagrangian method will be finally associated to a standard photosynthetic growth model to simulate batch cultivation in a torus PBR, retained here as a practical example. Although hydrodynamics will be introduced in the calculation, simulations will show that, without a dynamic interaction between photosynthetic conversion and fluctuating light regimes implied by cell movement along light gradient (the so-called light/dark cycles effects), PBR efficiency for a given species is only dependent on the light input and reactor geometry, according to the first principle of thermodynamics.
\end{abstract}

\section{Introduction}

In spite of the interest of photosynthetic microorganisms (algae, plant cells or photosynthetic bacteria) in various domains (Spolaore et al., 2006) industrial applications remain limited, mainly owing to the difficulty of proposing intensive cultivation systems with high biomass concentration and productivity. Light is known to be the principal limiting factor of photobioreactors (PBRs) efficiency (Richmond, 2004a,b). From the engineering point of view, in order to reach high culture densities, light input has to be increased, by working under high photons flux density (PFD), or by maximizing the illuminated surface for a given culture volume. Another possibility is to improve light conversion by photosynthetic cells, by cultivating specific strains, genetically modified or naturally adapted to the particular radiative transfer conditions encountered in PBRs (Kondo et al., 2006; Polle et al., 2002). But, because such an approach remains highly dependent on the species, it seems difficult to generalize. The present work aims at rigorouslv analvzing mixing conditions, which are supposed to influence light conversion, and thus PBRs efficiency, by modifying light access conditions.

Due to cell absorption and scattering, the radiation field is highly heterogeneous inside the culture. This makes PBR application different from other classical bioprocesses (fermentation in mixing tank for example), where mixing conditions are relevant to the homogenization of culture conditions by increasing mass and heat transfer. The assumption of a homogeneous distribution of chemical nutrients cannot be applied to light, whatever the mixing conditions. Flow effect is in the generated cell movement. By flowing in the heterogeneous radiation field, cells experience a particular history with respect to the light absorbed, composed of variations from high irradiance level (in the vicinity of the light source) to low or quasinull values (in the depth of culture) if biomass concentration is high. Influence on the resulting growth in PBR is still a problem to solve. Photosynthetic conversion is indeed a dynamic process, and the fluctuating light history induced by the flow can modify instantaneous conversion rate of absorbed light. This is the so called light-dark (L/D) cycle effect, widely described in the literature (cf. Richmond, 2004a or Janssen et al., 2000b for a review). But it is very difficult to experimentally investigate those effects in PBR, because of 
various mixing effects, like transfer enhancement (positive effect), shear-stress generation (negative effect)... Separating the coupling between the flow field and the light use from other possible mixing effects is difficult to achieve experimentally (Merchuk et al., 1998). In addition, L/D cycle effects are fully dependent on the light regime, and thus of cycles frequencies and magnitudes. In PBRs, such values are rarely known, the cell history with respect to light resulting from both flow and radiative fields, each being a problem on its own. For all of these reasons, flow conditions in PBRs are nowadays mainly empirically optimized.

Optimization of light use by modification of hydrodynamic conditions requires the formulation of two key-problems: knowledge of light history experienced by flowing cells, and the prediction of photosynthetic response to this history. If both are known, a modeling approach can be conducted to propose an innovative tool for hydrodynamic optimization of light use in PBRs. Modeling in a predictive way the photosynthetic response in dynamic light regime seems today unrealistic, the global response being the result of numerous possible interacting intracellular reactions, with various timescales, some of them being certainly unknown. This explains why only semi-empirical modeling approaches are usually conducted in the few examples devoted to this topic (Camacho et al., 2003; Luo and Al-Dahhan, 2004; Pahl-Wostl, 1992; Wu and Merchuk, 2001, 2002, 2004; Yoshimoto et al., 2005). On the contrary, the coupling between light transfer and cell displacement is predictable, each one being fully determined physically by radiative transfer theory and fluid dynamics.

Modeling of the biological cell transport in the reactor is important only if the nutrient concentration field cannot be assumed to be homogeneous. In PBRs, this is always the case for the light, but it also occurs with chemical nutrient in conventional bioreactor (BR) application as for example in large-scale fermentation processes, where homogeneous mixing is difficult to achieve, or when the environment of each cell becomes limited in a given growth substrate, as in high cell density cultures (Lapin et al., 2006; Larsson et al., 1996; Schmalzriedt et al., 2003). Bioreactor efficiency is then dependent on nutrient availability, which is the overall result of mixing conditions, nutrient concentration field and nutrient uptake. Both cells and nutrient concentration fields have to be known, to model the effect of mixing conditions on nutrient availability. By adding a local formulation of the growth kinetic and nutrient uptake, the effect of nutrient availability on the overall biological response in the BR can finally be represented.

The cell history in BR with respect to a particular nutrient can be formulated either in an Euler-Euler approach, or in an Euler-Lagrangian approach (see Lapin et al., 2006 for a review). Because living cells can often be considered as fluid elements (without slip velocity), an Euler-Euler approach seems attractive. Transport equations for both nutrient and living cells are then solved simultaneously. To formulate the coupling between abiotic and biotic phases, nutrient uptake is modeled in the transport equation of nutrient as a sink term dependent on the local concentration of living cells (Larsson et al., 1996). Such an approach is very similar to the case of reacting flow modeling (Fox, 2003). The only difference is in the formulation of the nutrient uptake rate, and of the corresponding biological response.

One limitation of assimilating a biological response to a simple chemical reaction is in its dependence on the physiological state, which is often the result of the cell life. In that case, the biological phase can be considered as composed of single elements, each having its own history. This is obtained using a Lagrangian approach. Trajectory of cells being known, their history with respect to the nutrient concentration can then be determined. Obviously, because the abiotic phase is not history dependent, an Eulerian approach can still be applied to the nutrient transport modeling. This Euler-Lagrange method was successfully applied by Lapin et al. (2006) in the BR case. The main drawback of the Lagrangian formulation is the increase in computational time, which explains why Eulerian formulation is more frequently used, but with an assumption that the transported phase is continuous and not history-dependent.

In the Lagrangian approach, because cells are considered as individual, an important number of cells have to be simulated to be representative of the overall population. Typically, a few thousand cells are usually simulated, which can be regarded as negligible compared to the usual concentration in BR of several millions of cells per milliliters. Results can however be considered as representative of the global response of the overall culture in most cases. Even with this great size reduction of the simulated population, computational time remains high. When considering living media, the problem of computational time is increased by the difference in characteristic timescales of biotic and abiotic phases. Typically, the doubling time of a photosynthetic cell is several hours, therefore a typical batch culture will last several days. Considering that trajectories are deduced from successive positions of the cell, this implies a calculation based on a very small time step $(<1 \mathrm{~s})$ to represent flow heterogeneity, the simulation of a complete bioprocess running requests long calculation time (several days of computing on a standard PC).

Owing to these limitations, the Lagrangian formulation of the biotic phase is mainly interesting for fundamental investigations. By determining the history of individual cells, the coupling with a biological model integrating effects of environmental fluctuations is then trivial. This facilitates the preliminary formulation of the model, and allows to analyze the relation between cell response and their environment modification. If the aim is a global optimization of the process, for example hydrodynamic optimization, it will be necessary to further simplify the Lagrangian formulation, for example by introducing statistical approaches. Comparison to predictions of the fully described Lagrangian model will be an important step in the model reduction.

Some examples can be found in the literature on the characterization of light regimes in PBRs, all based on the same approach. Firstly, cell trajectories are determined by using either a schematic representation of the flow (Janssen et al., 2003; Wu and Merchuk, 2002, 2004), by experimental measurement with radiative particle tracking (Luo and Al-Dahhan, 2004; Luo et al., 2003), or by a Lagrangian simulation (Perner-Nochta and Posten, 2007; Pruvost et al., 2002a,b; Rosello Sastre et al., 2007). Light regime is next obtained by introducing the light attenuation model, and by a simple projection of the cell trajectories on the radiation field. In those examples, flow and radiation fields are solved independently. This assumption seems accurate because of the specificity of both aspects, apparently decoupled from each other. This is verified for the flow field which is independent of the radiative heat transfer for non-thermal flows, which is easy to assume in PBRs that can be considered isothermal (if not the case, as in combustion systems, interactions can be very complex, as for example between turbulence and radiation: cf. Coelho, 2007 for a review). For the light transfer however, attention must be paid to the formulation of the coupling. Mixing can indeed influence the spatial distribution of particles participating in radiative transfer, which results in a modification of the radiation field (Cassano et al., 1995). This is well known in photoreactor investigations, where equations of momentum, mass transport and radiative transfer are solved simultaneously, as in photocatalysis (Pareek et al., 2003). Due to the high load and high density of catalytic particles, the solid phase has to be considered independent of the liquid phase. The increase in complexity when solving all transport equations simultaneously is moderated by the possibility to use an Euler-Euler approach for both fluid and solid phases transport, the catalytic reaction not being history-dependent. Ideally, such a method would have to be used in PBR application. But, as already explained, because of 
Light source (fluorescent tubes)

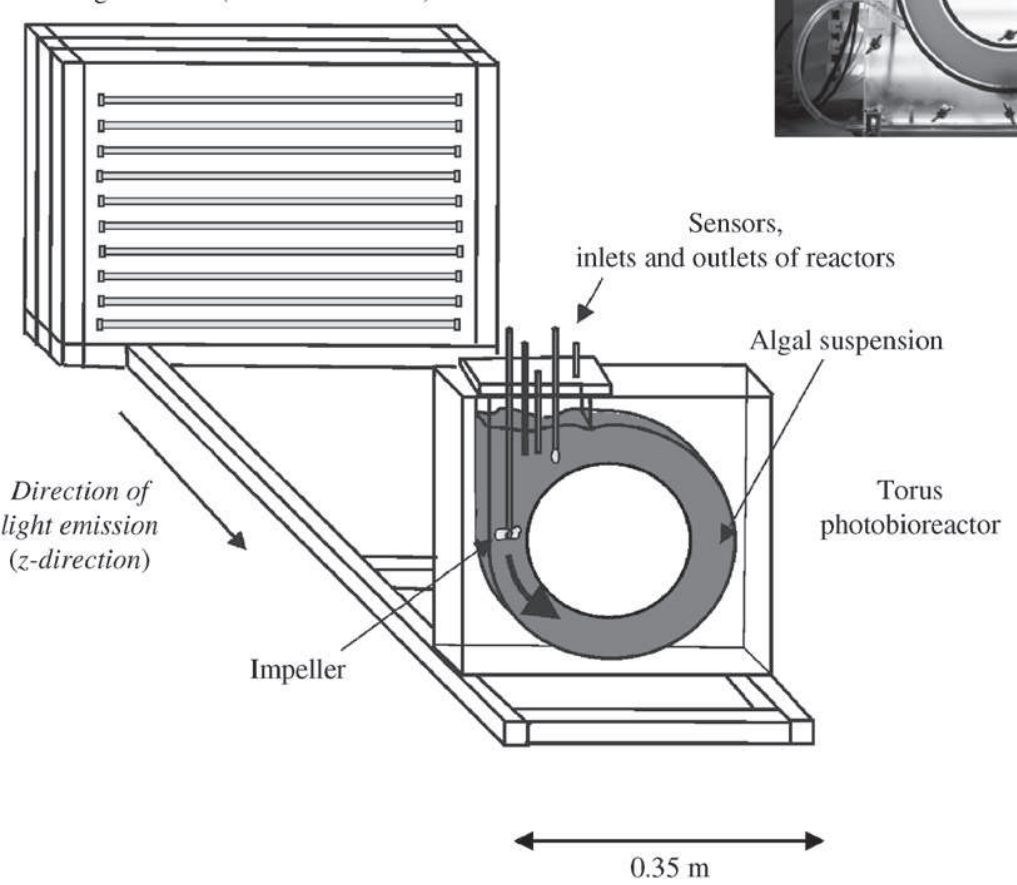

Fig. 1. Schematic representation and photography (front view) of the torus PBR

the possible existence of dynamic responses in the photosynthetic growth, a Lagrangian formulation is necessary for the biotic phase. A correct representation of the radiative transfer based on individual particle tracking increases however dramatically the population size to be simulated. This makes unrealistic to consider a global simulation of the PBR using such a method, which has to be simplified.

Transport of heavy particles in photocatalysis is for example more complex than cell transport in PBRs. Because of their very small size, with density close to that of water, photosynthetic cells can be considered as fluid elements. A mass transport equation is thus not necessary for flowing cells, only flow fields values being necessary. Another usual assumption is to suppose the biomass concentration in the reactor homogeneous. The consequence is that the radiative solution becomes independent of the mixing inside the reactor. To the authors' knowledge, such an assumption has always been applied when transport of a biological phase was considered, as for photosynthetic microorganisms cultivation (Luo and Al-Dahhan, 2004; Perner-Nochta and Posten, 2007; Wu and Merchuk, 2002, 2004), but also for UV disinfection (Elyasi and Taghipour, 2006). This was the case for example in previous authors' studies, which conclude with the interest of applying a swirling flow in an annular PBR to improve the light use in the cultivation of Porphyridium purpureum (Muller-Feuga et al., 2003a,b; Pruvost et al., 2002a).

In this work, it will be demonstrated that such a simple coupling cannot be made. Considering radiative transfer to be independent of mixing conditions will generate a methodic error in the Lagrangian formulation which introduces a non-existing influence of flow on the use of light. Calculation method of the radiative transfer will have to be modified to consider the effect of a non-ideal mixing. To avoid simulating a great amount of cells to represent their effects on light

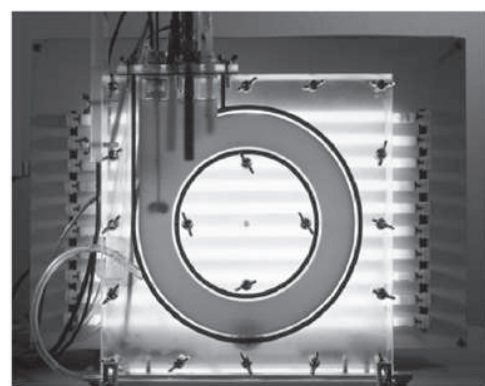

Sensors,

ets and outlets of reactors

Algal suspension

Torus

photobioreactor

attenuation in the culture, an original method will be presented, in accordance with the objective of a limited computation time to keep a formulation tractable for PBR modeling. Flow effect will be introduced in the radiative two-flux model, which proved to be efficient for PBR application, using a parameter representative of the heterogeneous residence time spent by flowing cells along the depth of culture. The proposed formulation will be demonstrated to be coherent with an energetic analysis of the PBR on both the photonic and material phases.

The validated Lagrangian formulation will be next applied for a theoretical investigation of a possible effect of flow on the use of light in PBRs. Two effects are indeed assumed. The first is the previously described L/D cycle effect, which includes various particular responses of photosynthetic microorganisms when submitted to light fluctuations, as when cells flow along the light gradient in PBRs. The second is a global effect induced by non-ideal mixing. Because of the light gradient, biological response is of the local type. Thus, if mixing modifies the residence time of flowing cells in given regions of the PBR, one can suppose an influence on the resulting growth in the PBR (Luo and Al-Dahhan, 2004). This is obvious that perfectly mixed conditions, and thus homogeneous residence time, are a theoretical hypothesis unachieved in practical case. So, this study will focus on the latter effect, because if it exists, it will be necessarily superposed to any L/D cycle effect in PBRs. For that purpose, Lagrangian formulation will be associated to a simple kinetics model of photosynthetic growth of Chlamydomonas reinhardtii. This will allow to simulate batch culture under non-ideal mixing conditions. The study will be conducted on a PBR of torus geometry (Fig. 1) where both flow and radiation fields have been extensively described elsewhere (Pottier et al., 2005; Pruvost et al., 2006). This reactor 
will serve as a practical example of application of the Lagrangian approach.

\section{Investigation of the coupling between flow conditions and light transfer using Lagrangian formulation}

\subsection{Light transfer modeling}

To simplify the investigation, only geometries responding to the Cartesian one-dimensional hypothesis will be investigated, but all following conclusions can be extended to the general case of a multi-directional attenuation with a little adaptation effort. If the rectangular one-dimensional hypothesis is assumed for radiative transfer, light attenuation occurs along only one main direction (named the depth of culture, represented by $z$ ), perpendicular to the illuminated surface of the PBR. This hypothesis allows simplified radiative model to be used, and analytical solutions of the irradiance field to be obtained. For example, the two-flux model revealed to be well adapted to the case of photosynthetic microorganisms cultivation, where both absorption and scattering of light occurs (Cornet et al., 1995, 1998), mainly in the usual case of quasi-collimated incidence (Pottier, 2005).

Considering a normal incidence and a quasi-collimated field of radiation, the two-flux model is characterized by the following set of differential equations as a simplification of the full photon transport equation (Cassano et al., 1995) (to simplify calculations, the two-flux model is not presented in its wavelength-dependent form, but the method can be easily extended to a spectral resolution. See Pottier et al., 2005 for details):

$\left\{\begin{array}{l}\frac{\mathrm{d} I^{+}}{\mathrm{d} z}=-E_{a} X I^{+}-b E_{S} X\left(I^{+}-I^{-}\right) \\ \frac{\mathrm{d} I^{-}}{\mathrm{d} z}=E_{a} X I^{-}-b E_{S} X\left(I^{+}-I^{-}\right)\end{array}\right.$

$E_{a}$ and $E_{S}$ are, respectively, the mass absorption and scattering coefficients of light of photosynthetic microorganisms, $b$ the backward scattering fraction, and $X$ the biomass concentration in the culture medium. $I^{+}$and $I^{-}$are the specific radiant intensities in forward and backward directions, respectively.

Resolution with appropriate boundary conditions gives the irradiance field inside the culture:

$G(z)=I^{+}+I^{-}$

where $G(z)$ is the irradiance at a given depth of culture $z$.

Boundary conditions depend on the illumination conditions and reactor geometry. The reactor usually presents a front transparent surface for illumination, the back side being fully transparent or semi-reflective. This can be represented by introducing the reflection coefficient $\rho$, with $\rho=0$ in the fully transparent case, and $\rho=1$ for total reflection. This gives the following boundary conditions:

$\begin{cases}z=0 & I^{+}=q_{0}^{+} \\ z=L & I^{-}=\rho I_{L}^{+}\end{cases}$

where $q_{0}^{+}=q_{\cap}$ is the hemispherical incident light flux (or PFD as commonly named in PBR studies) and $L$ the light path length (reactor depth).

The torus PBR can be used even with a back side in stainless steel $(\rho=0.3)$ for temperature regulation purpose, or with a fully transparent back side $(\rho=0)$. For simplification, only the latter will be investigated (corresponding to the set-up of Fig. 1). The irradiance profile is thus given by

$\frac{G(z)}{q_{\cap}}=2 \frac{(\alpha+1) \exp [\delta(L-z)]+(\alpha-1) \exp [-\delta(L-z)]}{(\alpha+1)^{2} \exp (\delta L)-(\alpha-1)^{2} \exp (-\delta L)}$

with the two-flux extinction coefficient $\delta=X \sqrt{E_{a}\left(E_{a}+2 b E_{S}\right)}$ and the linear scattering modulus $\alpha=\sqrt{E_{a} /\left(E_{a}+2 b E_{s}\right)}$.
Eq. (4) is valid only if the biomass concentration $X$ is taken as homogeneous along the $z$-direction. As shown later, if not the case, a numerical resolution of the representative differential equations of the two-flux model (Eq. (1)) will be necessary.

Because its optical properties have been well determined in a previous work (Pottier et al., 2005), Chlamydomonas reinhardtii 137c was retained for the study. Corresponding radiative coefficients are then calculated by the generalized Lorenz-Mie theory as

$E_{a}=172 \mathrm{~m}^{2} / \mathrm{kg}, \quad E_{S}=868 \mathrm{~m}^{2} / \mathrm{kg}, \quad b=0.01728$

It must be noticed that an extensive comparison of the theoretical one-dimensional irradiance attenuation profiles was done elsewhere (Pottier, 2005) in the same PBR, showing a very good agreement with experimental results.

\subsection{Lagrangian determination of cell trajectories}

Cell trajectories can be evaluated using a Lagrangian formulation. A full description and its validation can be found in Pruvost et al. (2002b). Only main features are presented here, and especially the slight modification in equation formulation when using computational fluids dynamics (CFD) results. Indeed trajectory calculation assumes the velocity-field is known, either by an experimental characterization (Pruvost et al., 2000), or by numerical prediction. In the field of flow condition optimization, CFD appears the most interesting. A previous work has shown the interest of a commercial code (such as Fluent ${ }^{\circledR}$ ) to obtain accurate flow predictions in a PBR of torus geometry (Pruvost et al., 2006). This PBR will serve as a practical example in the remaining part of this study.

If it is considered that microalgae have the same density as the fluid, any mass effect can be neglected. Correction of pathlines when particles are of greater size than the smallest flow eddies are not considered either, because of the cells size which is about $10 \mu \mathrm{m}$ and thus smaller than the Kolmogorov scale. These two assumptions consider microalgae as a passive tracer represented by elementary fluid particles (or "notional" particles, as described by Fox, 2003) in trajectory calculation. Trajectories are then obtained step by step by calculating the successive positions $P$ of a fluid element by using:

$P(t+\Delta t)=P(t)+U_{P} \Delta t$

where $U_{P}$ is the instantaneous velocity at a given position $P$ and $\Delta t$ a time step to specify.

$\Delta t$ is set sufficiently small to have no influence on the resulting trajectories ( $\Delta t=0.01 \mathrm{~s}$ in the case of the torus PBR). Eq. (5) is fully determined in laminar regime, but in turbulent regime, due to the fluctuating nature of the velocity, a specific formulation is requested. The Reynolds decomposition of instantaneous velocity can be applied. The instantaneous velocity at a given position $P$ is then:

$U_{P}=\overline{U_{P}}+u_{P}^{\prime}$

where $\overline{U_{P}}$ is the mean value of velocity and $u_{P}^{\prime}$ the fluctuating one.

Turbulent models based on Reynolds time-averaged Navier-Stokes (RANS) equations give a good compromise between CPU effort and prediction accuracy in practical case simulations. But CFD results are not sufficient to solve Eq. (6), because only mean values (velocity and turbulent characteristics) are given by RANS equation resolution. The fluctuating part remains to be formulated, in relation to turbulent flow characteristics. Fluctuating part of the velocity is determined using a stochastic model in which $u_{P}^{\prime}$ is not considered as purely random, but is also a function of turbulence correlations (to represent the spatial structure of eddies). A Langevin formulation is used, in which the fluctuating part $u_{P}^{\prime}$ is divided in two terms, the first being a pure random function $e$ and the second linked to the structured nature of turbulence (Pruvost et al., 2002b). Because 
of turbulent spatial correlations, fluctuating velocity in Eq. (6) at a given position $P(t)$ is thus a function of the previous one $P(t-\Delta t)$

$u_{P(t)}^{\prime}=f u_{P(t-\Delta t)}^{\prime}+g e_{P(t)}$

where $e$ is a Gaussian distributed random function, with its root mean square equal to turbulence intensity $U^{\prime} . f$ and $g$ are weighting parameters linked, respectively, to the correlation and random terms, with $f=\exp \left(-\left(r / L_{e}\right)^{2}\right)$ and $g=\sqrt{1-f}$.

$L_{e}$ is the integral length scale of turbulence, and $r$, the distance traveled from an initial position. Obviously, term $(f)$ tends to 0 and term $(g)$ to 1 when $r$ increases. In other words, when going away from the initial position, the correlation between values determined at position $P$ and at the initial position tends to vanish. A maximum length $L_{C}$ is thus defined and when $r>L_{C}$, the current position of the trajectory is redefined as the new initial point for the spatial correlation in Eq. (7). This allows to frequently recalculate the correlation term $(f)$ and to have a good representation of the influence of the structured nature of turbulence on trajectories. $L_{C}$ is defined equal to $L_{e} / 2$ (Pruvost et al., 2002b). In duct flows the integral length scale determined along perpendicular directions of the flow is around half value of the one along main direction (Hinze, 1975). If velocity components other than the axial one (in the main direction of the flow) are considered, correlation term ( $f$ ) will be given by

$f=\exp \left(-\left(2 r / L_{e}\right)^{2}\right)$

All the local hydrodynamic values necessary for the cell trajectory calculation in the torus PBR have been obtained using CFD, the turbulent flow field being solved with a $k-\omega$ model (based on RANS equations). Such resolution gives the field of values for mean components of velocity, turbulent kinetic energy $k$ and turbulent viscosity $\mu_{t}$ (deduced from the Boussinesq hypothesis). Local values necessary for the fluctuating velocity formulation (Eq. (7)) are thus obtained using following relations (isotropic turbulence is assumed in the $k-\omega$ model):

$U^{\prime}=\left(\frac{2}{3} k\right)^{1 / 2}$ and $L_{e}=\frac{\left(\frac{2}{3} k\right)^{3 / 2}}{\varepsilon} \quad$ with $\varepsilon=\frac{\rho_{f} C_{\mu} k^{2}}{\mu_{t}}$

$\rho_{f}$ being the fluid density and $C_{\mu}=0.09$ ( $k-\omega$ model constant).

\subsection{Integration of the radiative model in the Lagrangian approach}

\subsubsection{Direct coupling of the radiative model with the Lagrangian approach}

The investigation was conducted in the PBR of torus geometry, both light transfer and hydrodynamic aspects being already described in previous authors' works (Pottier et al., 2005; Pruvost et al., 2006). An example of cell trajectories is given in Fig. 2 for a given impeller rotation speed $N_{\text {imp }}$ which determines hydrodynamic conditions inside the PBR (corresponding Reynolds number based on the mean bulk velocity $U_{0}$ is given, with $R e=U_{0} L / v$ : see Pruvost et al., 2006 for calculation details. Reynolds number for each value of $N_{\text {imp }}$ investigated are also given in Table 1). By projecting trajectory along the depth of culture, and by using Eq. (4) to calculate the irradiance for each successive position, light history can be easily determined. It must be noticed that such a method, where both cell trajectories and light attenuation model are directly coupled, is always applied in the few works devoted to the investigation of light regimes in PBR. An example of results given in Fig. 3 confirms that flowing cells are submitted to a complex fluctuating light regime induced by flow conditions, each cell experiencing fast irradiance fluctuations composed of fully illuminated periods when the cell is in the vicinity of the light source, and dark periods in the depth of culture. This
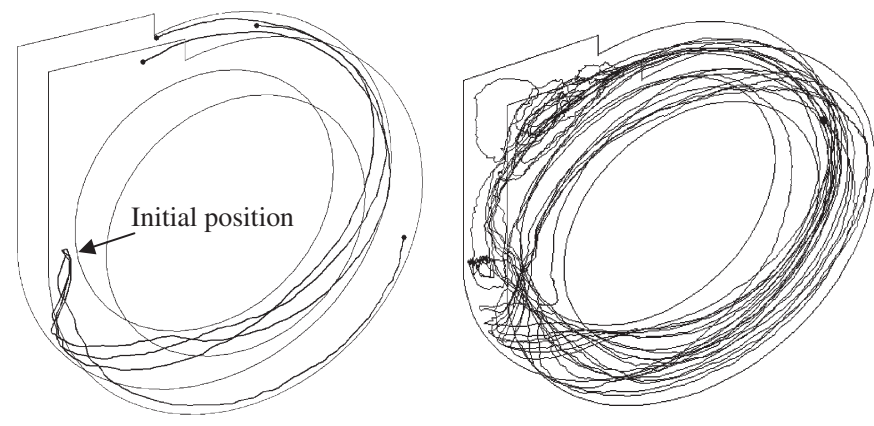

Fig. 2. Example of cell trajectories determined using the Lagrangian approach. Left: Number of cells simulated $=4-$ Time course of trajectories $=4 \mathrm{~s}-N_{\mathrm{imp}}=250 \mathrm{rpm}$ $(R e=3700)$ Right: Number of cells simulated $=1-$ Time course of trajectories $=300 \mathrm{~s}$ $-N_{\text {imp }}=250 \mathrm{rpm}(R e=3700)$.

Table 1

Examples of results of the mean light availability for various absorbing conditions inside the torus PBR

\begin{tabular}{llllll}
\hline & $\begin{array}{l}N=300 \mathrm{rpm} \\
(R e=4500) \\
X=0.5 \mathrm{~g} / \mathrm{l}\end{array}$ & $\begin{array}{l}N=300 \mathrm{rpm} \\
(R e=4500)\end{array}$ & $\begin{array}{l}N=300 \mathrm{rpm} \\
(R e=4500)\end{array}$ & $\begin{array}{l}N=250 \mathrm{rpm} \\
(R e=3700)\end{array}$ & $\begin{array}{l}N=400 \mathrm{rpm} \\
(R e=6500)\end{array}$ \\
& & $X=1.2 \mathrm{~g} / \mathrm{l}$ & $X=1.2 \mathrm{~g} / \mathrm{l}$ & $X=1.2 \mathrm{~g} / \mathrm{l}$ \\
\hline$\langle G\rangle \mu \mathrm{E} / \mathrm{m}^{2} \mathrm{~s}$ & 63 & 35 & 29 & 29 & 29 \\
$\bar{G}_{\infty} \mu \mathrm{E} / \mathrm{m}^{2} \mathrm{~s}$ & 73 & 46 & 41 & 45 & 47 \\
$\left\langle G_{h}\right\rangle \mu \mathrm{E} / \mathrm{m}^{2} \mathrm{~s}$ & 73 & 45 & 40 & 44 & 47 \\
\hline
\end{tabular}

Comparison between the different methods of calculation.

is a well known result (Perner-Nochta and Posten, 2007). The keyproblem is to formulate possible effects of flow conditions on the light conversion in biomass inside the reactor, which remain hard to accurately quantify in practice, although this is a subject of numerous works, either on experimental characterization (Janssen et al., $2000 \mathrm{~b}$ ) or on establishment of dynamic models for photosynthetic conversion (Camacho et al., 2003; Eilers and Peeters, 1993; Luo and Al-Dahhan, 2004; Pahl-Wostl, 1992; Wu and Merchuk, 2001, 2002, 2004; Yoshimoto et al., 2005; Zonneveld, 1998). The present study was voluntarily focused on the other presumed hydrodynamics effect that is a modification of the mean light availability in the culture due to non-ideal mixing conditions (global effect). Before investigating the dynamic kinetic effect, it is necessary to conclude from the first: if a dynamic effect exists, it will necessarily integrate the global effect.

To represent the global effect which only deals with the mean light available in the culture, it is interesting to compare values obtained by the Lagrangian approach which includes flow effect, with those calculated by a simple spatial integration of the radiation field achieved inside the reactor. For the Lagrangian approach, the mean amount of light is determined by averaging on a period $T$ instantaneous irradiances $G(t)$ available for each successive location of a flowing cell $i$ :

$\overline{G_{i}}(T)=\frac{1}{T} \int_{0}^{T} G_{i}(t) d t$

To obtain a value representative of the whole population, it is necessary to repeat calculation on a large set of $N$ individual cells. $\bar{G}(T)$ represents thus the average amount of light available in the culture ( = denotes a time averaging):

$\bar{G}(T)=\lim _{N \rightarrow \alpha}\left(\frac{1}{N} \sum_{i=1}^{N} \overline{G_{i}}(T)\right)$ 

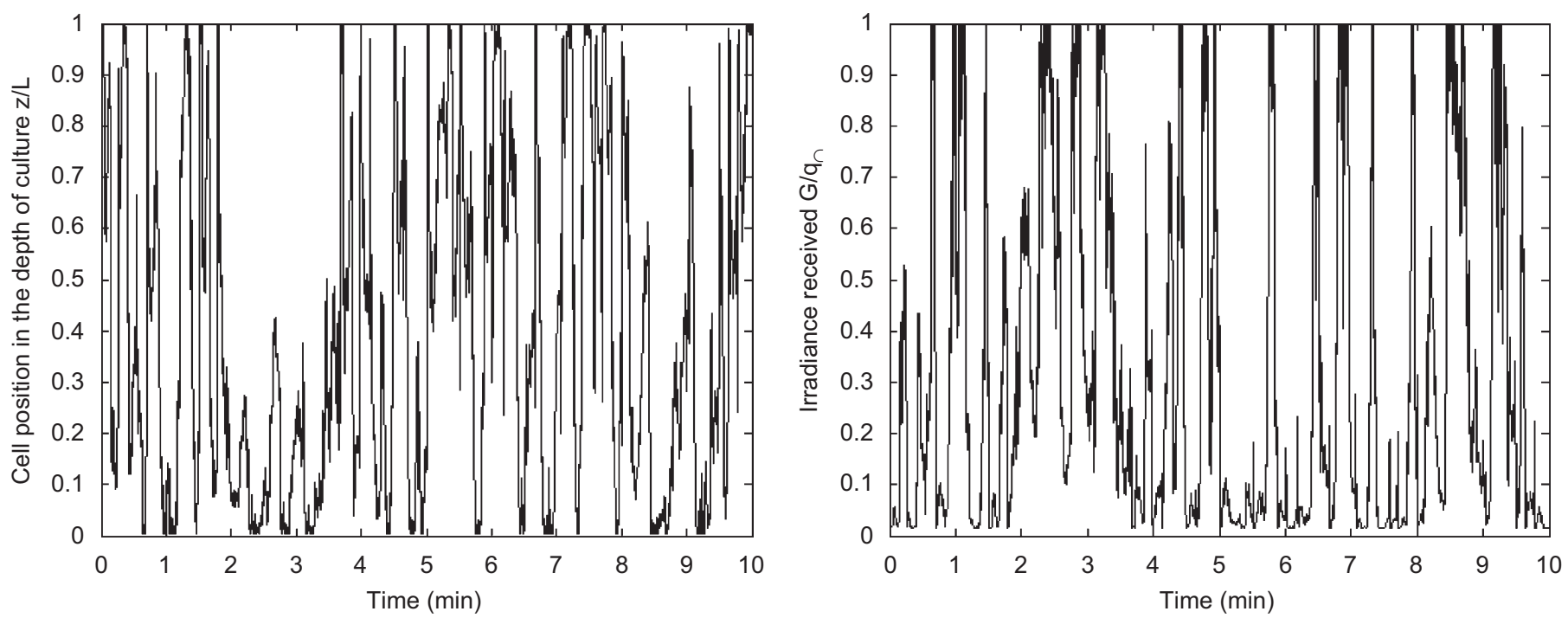

Fig. 3. Example of cell displacement along the light gradient (left) and of corresponding light regime (right) encountered in the torus PBR $\left(N_{\text {imp }}=300 \mathrm{rpm}-X=1 \mathrm{~g} / \mathrm{l}-q_{\cap}=200 \mu \mathrm{E} \mathrm{m}^{-2} \mathrm{~s}^{-1}\right)$.

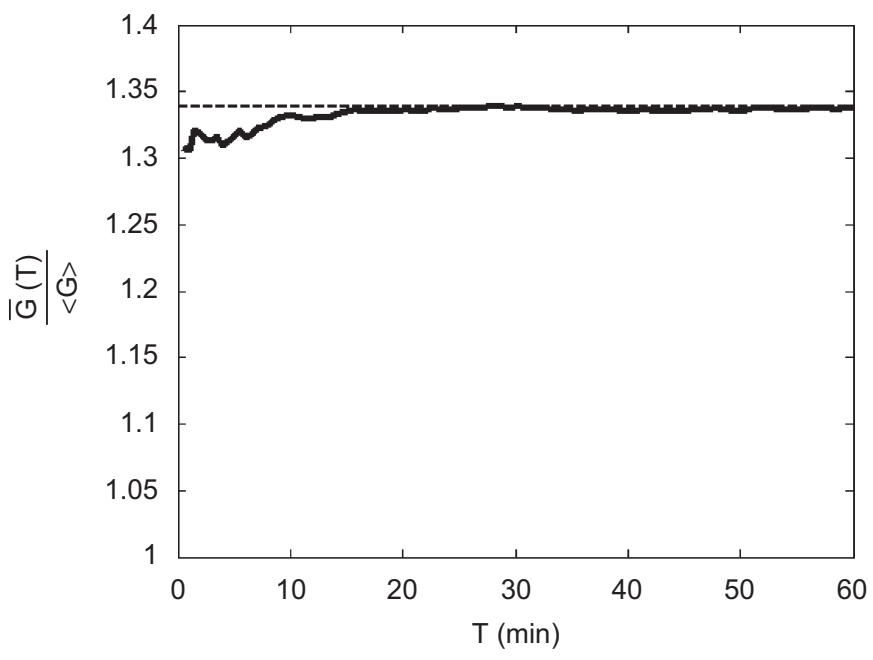

Fig. 4. Mean available light in the culture as a function of time course inside the reactor. Comparison between spatial and temporal averaging $\left(N_{\text {imp }}=300 \mathrm{rpm}-X=1 \mathrm{~g} / \mathrm{l}-q_{\cap}=200 \mu \mathrm{E} \mathrm{m}^{-2} \mathrm{~s}^{-1}\right)$.

This value can be compared to the one obtained by integrating the irradiance field:

$\langle G\rangle=\frac{1}{V} \int_{0}^{V} G(V) \mathrm{d} V$

In the case of a one-dimensional attenuation, we obtain $(\langle\rangle$ denotes a spatial averaging):

$\langle G\rangle=\frac{1}{L} \int_{0}^{L} G(z) d z$

The ratio of values calculated with the two calculation methods (Eqs. (11) and (13)) is presented in Fig. 4, as a function of the exposure period $T$ and for same radiative transfer conditions (similar values of $X$ and $q_{\cap}$ ). Results for the Lagrangian approach have been obtained for $N=800$ cells, which revealed to be sufficient to reach independence with $N$ in the calculation of $\bar{G}(T)$. After a long illumination period $T$, a constant value of the light available is achieved. This value is denoted
$\overline{G_{\infty}}$ and thus represents the average amount of light received by the culture after an infinite period of circulation:

$$
\overline{G_{\infty}}=\lim _{T \rightarrow \infty}(\bar{G}(T))
$$

In the conditions investigated, the value of $\langle G\rangle$ is $34.8 \mu \mathrm{E} \mathrm{m}^{-2} \mathrm{~s}^{-1}$ the one obtained by temporal averaging $\overline{G_{\infty}}$ being $46.6 \mu \mathrm{E} \mathrm{m}^{-2} \mathrm{~s}^{-1}$ (in the following, results are given in $\mu \mathrm{E} \mathrm{m}^{-2} \mathrm{~s}^{-1}$, a unit commonly used in PBR studies. For the daylight spectrum used by photosynthesis, $4.6 \mu \mathrm{E} \mathrm{m}^{-2} \mathrm{~s}^{-1} \cong 1 \mathrm{~W} \mathrm{~m}^{-2}$. See Notation for details). Extension to the simulation of other operating conditions (radiative transfer and flows conditions) leads to same tendencies with a difference in results for both calculations methods (data not shown). Because only temporal averaging considers cell trajectories, hence flow influence, such a result contributes to the idea of a possible global effect of flow on the light transfer, by modifying the amount of light received by cells (around 30\% higher in the case on the torus reactor, depending on the conditions tested). A similar result was obtained by Luo and Al-Dahhan (2004), who investigated the effect of cell trajectories on light availability, by comparing both spatial and temporal averagings. This result appears interesting, because if flow conditions could increase the amount of available light, the light limitation in PBR would be reduced leading to higher PBR efficiency. This also explains results of a previous work by the authors (Pruvost et al., 2002a), where interest of applying a swirling flow to increase biomass concentration in an annular photobioreactor was emphasized. Unfortunately, as can be proved by an energetic approach of light energy conversion in PBR (see Appendix A), such a conclusion is not valid. The problem comes from the fact that the mean available energy $\langle G\rangle$ or $\overline{G_{\infty}}$ does not represent the right "physical" quantities to be used rigorously in formulating the kinetic coupling in PBR. As already well documented indeed (Cassano et al., 1995; Cornet et al., 2001, 2003), the local volumetric rate of radiant energy absorbed (LVREA) $A=a G$ inside the reactor, appearing theoretically when writing energy balances on the PBR (Appendix A), enables to distinguish between the available energy (the irradiance $G$ ) from the photonic phase, and the actual rate of radiant energy absorbed by the material phase (the cells) and used for photosensitized reactions. The minor (but not negligible) difference arises in the use of the volumetric absorption coefficient $\left(a=E_{a} X\right.$ ) which is a typical property of the material phase and represents the probability for any photon 


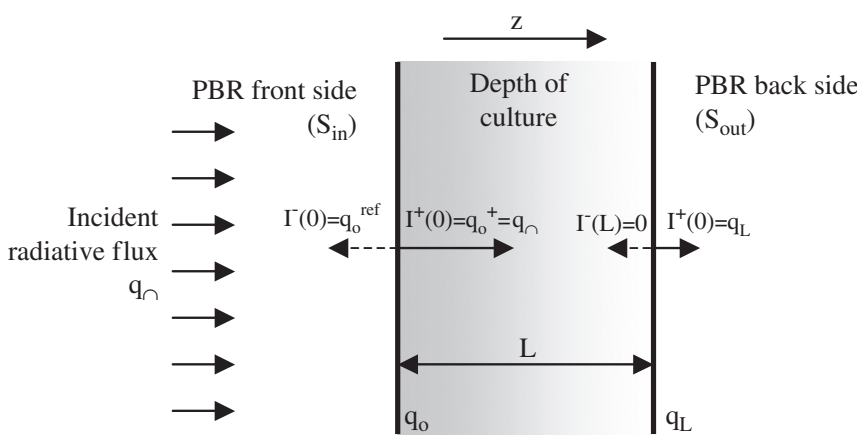

Fig. 5. Boundary conditions for the radiative transfer inside the torus PBR.

to be absorbed in a collision with a cell (Appendix A). At this stage of our reasoning, because homogeneous radiative properties for the material phase are considered (constant $a$ and $s$ in space leading to a linear radiative transfer problem), there is no problem in defining the mean rate of radiant energy absorbed (MVREA) using $\langle A\rangle$ instead of $\langle G\rangle$ just multiplying by the constant $a$ :

$\langle A\rangle=\frac{1}{V} \int_{V} A \mathrm{~d} V=\frac{1}{V} \int_{V} a G \mathrm{~d} V=\frac{1}{V} \int_{V} E_{a} X G \mathrm{~d} V$

Eq. (15) can be rewritten in the case of one-dimensional attenuation and by assuming a homogeneous concentration inside the reactor:

$$
\begin{aligned}
\langle A\rangle & =\frac{1}{L} \int_{0}^{L} E_{a} X(z) G(z) \mathrm{d} z=\frac{1}{L} \int_{0}^{L} a(z) G(z) \mathrm{d} z \\
& =\frac{a}{L} \int_{0}^{L} G(z) \mathrm{d} z=a\langle G\rangle=E_{a} X\langle G\rangle
\end{aligned}
$$

In the same manner, with no more difficulties, one obtains for the asymptotic dynamic MVREA taking into account Lagrangian trajectories after a long period of time:

$\overline{A_{\infty}}=\lim _{T \rightarrow \infty}(a \bar{G}(T))=a \overline{G_{\infty}}$

Additionally, as demonstrated in Appendix A, energetic balances on the photonic and material phases imply that the total amount of energy absorbed by the culture was also fixed by the radiative flux input and output on the external surfaces of the reactor (see Fig. 5). As a consequence, the MVREA may be obtained either by averaging the LVREA on the material phase volume or by vectorial summation from the boundary conditions in PFD from (see Appendix A):

$\langle A\rangle=\frac{1}{L} \int_{0}^{L} a G \mathrm{~d} z=\frac{1}{V}\left[S_{0}\left(q_{0}-q_{L}\right)\right]=\frac{1}{L}\left(q_{0}-q_{L}\right)$

where $S_{0}$ is the PBR illuminated surface.

This equation shows that mixing conditions cannot modify the mean amount of light absorbed by the culture. This is especially obvious for high absorbing conditions, where light is fully absorbed when crossing the PBR $\left(q_{L}=0\right)$. The total radiative energy absorbed is thus only dependent on flux on illuminated surface, and thus not flow dependent. To verify the energetic balance, i.e., the first principle of thermodynamics, Lagrangian approach and spatial averaging must therefore give the same result, i.e.:

$\langle A\rangle=\overline{A_{\infty}}$

This result clearly demonstrates that it is not possible to improve the performances of any PBR by a primary coupling between hydrodynamics (taking into account cell trajectories history) and radiation field. The difference observed previously confirms that a direct linear coupling between flow and radiative transfer in the Lagrangian formulation is not correct.

\subsubsection{Formulation of mixing influence in the radiative model}

Ideally, the radiative transfer being dependent on the absorbing species spatial distribution, both mass transport and radiative model equations have to be solved simultaneously if non-ideal mixing conditions are applied, the resulting concentration field not being homogeneous. Such an approach is usually conducted in photoreactors (Cassano et al., 1995). But, as already explained, because photochemical species are not history dependent, the Lagrangian formulation is not necessary, allowing the Euler-Euler approach to be applied. If the absorbing phase transport is solved with a Lagrangian approach, it is necessary to solve thousands of trajectories simultaneously to have a representation of the concentration field in the reactor, hence of the resulting radiation field (even in this case, a correct representation is difficult, as discussed further). Such a method seems unrealistic for PBR application. As shown in the final part, the simulation of few cells in batch culture requests several days of calculation on a standard personal computer (PC).

However, the particular case of PBR allows a simplification, compared to the modeling of photocatalytic processes. Photoreactors are indeed generally operated with high load of heavy photocatalytic particles. Interaction of fluid and solid phases with respect to the flow field is therefore complex (Cassano et al., 1995; Pareek et al., 2003). As already explained, for photosynthetic cells, because of their small concentration (compared to photocatalysis) and density near unity, the culture can be easily assumed as a passive tracer. The coupling between momentum and mass transport equations in the photocatalysis case can thus be neglected in PBR applications. This means that the flow field can be solved independently of mass transport and radiative-transfer equations. Considering that the flow field has been previously determined (by CFD for example), the remaining problem is to formulate accurately how it could affect the resulting radiative-transfer. With an Eulerian approach, the problem is trivial, because solving mass transport of absorbing species gives directly its concentration field for given hydrodynamics conditions, which can be associated next in radiative model. In the Lagrangian formulation, it is less obvious. The concentration field must be ideally deduced from trajectories of individual cells. But this is clearly a weakness of Lagrangian approach. Even in the case of solving thousands of trajectories, concentration field remains difficult to accurately predict, especially because of the difficulty to account for mass diffusion which depends on concentration gradient better represented in an Eulerian framework. In Lagrangian framework, this implies more advanced and CPU time consuming methods (see Fox for examples). These methods are justified in the case of modeling reacting flows for example, where local reaction rates are highly dependent on local concentrations of reacting species for example (it is the same for BR applications, if effect of an heterogeneous nutrient concentration has to be modeled, see Lapin et al., 2006). Fortunately, such kind of coupling can be neglected in first instance in PBRs case. Radiative energy is indeed not a flow-transported property (like nutrients), and characteristic time of biomass concentration evolution has several orders of magnitude higher than homogenization-mixing time (biomass concentration can thus be regarded as a constant during absorption process). However, as shown before, it remains to correctly formulate effect of non-ideal light access conditions (mixing) to the light availability when using Lagrangian formulation.

Firstly, temporal information given by the Lagrangian approach has to be linked to a spatial dependent value. This will allow its relation to radiative transfer resolution that is only spatial dependent. This is obtained by further investigating the difference in mean light absorbed as previously observed between the Lagrangian approach and the spatial averaging of irradiance profile. This can indeed only be explained by a modification of light access due to the flow. Because the reactor is not perfectly mixed, cells can have various residence times along the depth of culture. This is shown in Fig. 6, where 


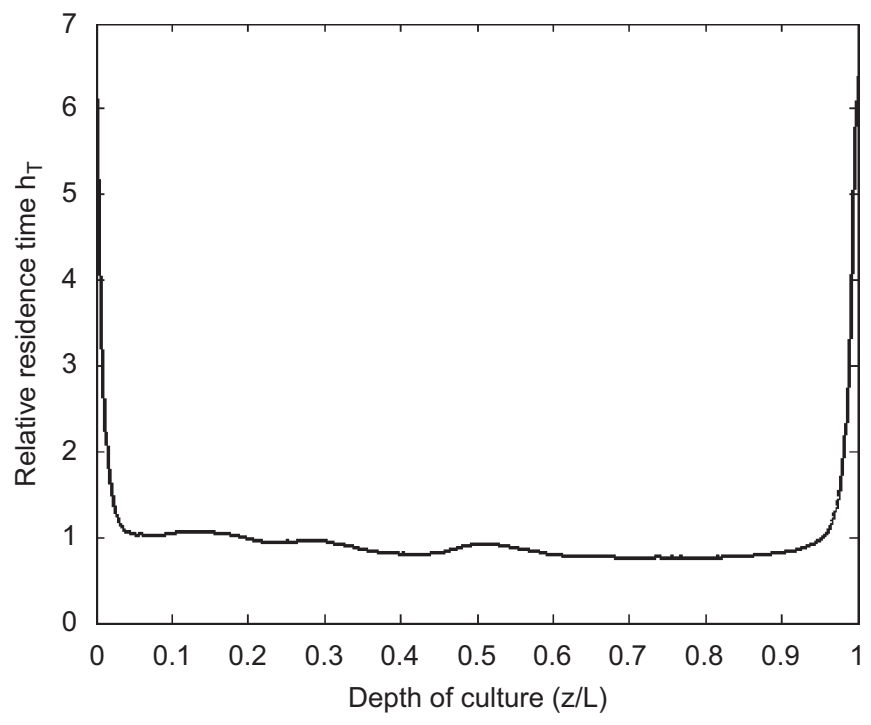

Fig. 6. $z$-RTD in the torus PBR for $N_{\text {imp }}=300 \mathrm{rpm}$.

cell trajectories are used to calculate the residence time distribution (RTD) of flowing cells with respect to the direction of light penetration ( $z$-direction). The RTD (named $z$-RTD in the following) has been normalized to give a relative residence time named $h_{T}(z)$, defined as follows:

$h_{T}(z)=\frac{H(z)}{\int_{0}^{L} H(z) \mathrm{d} z}$

where $H(z)$ is the $z$-RTD (histogram of the time spent at a given depth of culture, deduced from 800 cell trajectories to have a stable distribution).

The $h_{T}(z)$ distribution obtained in the torus PBR is almost homogeneous (for about $96 \%$ of the depth of culture) except near walls, where the residence time greatly increases. In the Lagrangian point of view, this indicates that flowing cells have flow patterns that lead to an heterogeneous residence time along $z$-direction. Obviously, in the case of perfectly mixed conditions (no preferential flow patterns), a uniform RTD will be achieved (in that case, we will obtain trivially $\left.h_{T}(z)=1\right)$. Thus, the $h_{T}(z)$ distribution can be regarded also as the difference between ideal and non-ideal mixing conditions with respect to light access. Because the spatial averaging implicitly supposes no flow influence, by extension, $h_{T}(z)$ can be used to represent the difference between spatial and temporal averaging. To illustrate this, it is interesting to introduce the $h_{T}(z)$ distribution in the spatial averaging of the available light, by weighting local values of irradiance in the integration. The new value of the mean amount of available light is noted $\left\langle G_{h}\right\rangle$, and is defined by

$\left\langle G_{h}\right\rangle=\frac{1}{L} \int_{0}^{L} h_{T}(z) G(z) \mathrm{d} z$

Some examples of results are given in Table 1 , for different absorbing and hydrodynamics conditions. When $h_{T}(z)$ is introduced, the same results are achieved as with the temporal averaging of the Lagrangian approach $\left(\overline{G_{\infty}}\right)$, demonstrating the ability of the proposed approach to obtain a good description of the hydrodynamics in saving calculation time. At this stage however, the differences obtained between the two spatial-averaged MVREA (with or without the $h_{T}(z)$ function) are still inconsistent with the first principle of thermodynamics. But, considering results obtained while introducing $h_{T}(z)$, such value appears suitable to clear off this inconsistency.

This can be achieved considering rates of absorption. Because a non-uniform $z$-RTD means that a cell will spend different times at a given depth of culture, mean local rates of absorption have thus to be modified with respect to time, proportionally to $h_{T}(z)$. This assumption is justified by the specific nature of radiative transfer (an exchange between a photonic and a material phase), the local photon absorption rate being a source term for the material phase (and a sink term for the photonic phase): as soon as photons are absorbed by cells at a given abscissa, they are no more available for the remaining part of the reactor. So, if cells spend statistically more time in a given zone, local absorption rates will be increased proportionally (an analogy to heat flux absorption can be conducted here, assuming no exchange between heated molecules. If the case, temperature will locally increased near a heated wall for example). This absorption rate being characterized by the volumetric absorption coefficient $a=E_{a} X$, if this value is weighed by $h_{T}(z)$ due a non-uniform residence time, we obtain a local formulation defined by

$a(z)=h_{T}(z) E_{a} X$

Because, as an intrinsic radiative property of each cell, $E_{a}$ is constant, it is natural to relate $h_{T}(z)$ with $X . h_{T}(z)$ can therefore be regarded as introducing a pseudo heterogeneous concentration field inside the reactor the distribution of which along the depth of culture will be given by

$X(z)=h_{T}(z) X$

where $X$ is the mean biomass concentration inside the reactor.

This equation is a Eulerian transcription of how absorption rates can be modified when residence time along the light gradient is not homogeneous. In the Lagrangian approach, a non-uniform $z$-RTD represents a heterogeneous time of exposure at a given depth of culture. It results in a modification of local absorption rates proportionally to $h_{T}(z)$, which is the same, following Eq. (23) and therefore taking a Eulerian representation, as to introduce a pseudo heterogeneous concentration field of absorbing species (introduction of "pseudo" term is explained here by the weakness of Lagrangian approach to predict the exact concentration-field, as discussed before). Despite a difference in its interpretation, the value $h_{T}(z)$ can thus be applied for both approaches. If mixing is supposed to affect local absorption rates, the final step is its introduction in the radiative transfer modeling. Because Eq. (4) supposes volumetric absorption coefficient is homogeneous, it is necessary to modify constitutive equations of the two-flux radiative transfer model, by introducing Eq. (23) as a well known non-linear definition of the optical thickness in the set of ordinary differential equations:

$$
\left\{\begin{array}{c}
\frac{\mathrm{d} I^{+}}{\mathrm{d} z}=-E_{a} X(z) I^{+}-b E_{S} X(z)\left(I^{+}-I^{-}\right) \\
=-E_{a} h_{T}(z) X I^{+}-b E_{S} h_{T}(z) X\left(I^{+}-I^{-}\right) \\
\frac{\mathrm{d} I^{-}}{\mathrm{d} z}=E_{a} X(z) I^{-}-b E_{S} X(z)\left(I^{+}-I^{-}\right) \\
=E_{a} h_{T}(z) X I^{-}-b E_{S} h_{T}(z) X\left(I^{+}-I^{-}\right)
\end{array}\right.
$$

If $h_{T}(z)$ and boundary conditions are known, such a system can be numerically solved (function bvp4c in Matlab ${ }^{\circledR}$ software), giving the irradiance field in the reactor. An example of attenuation profile is given in Fig. 7. Introduction of a heterogeneous residence time along the depth of culture modifies the irradiance field inside the reactor, especially near the optical surface, where the high value of residence time increases the absorption, i.e., attenuation. On the contrary, because all the radiative energy is rapidly absorbed, less influence of $h_{T}(z)$ is observed in the reactor depth, emphasizing the non-linear relationship between the non-uniform $z$-RTD and the light transfer. In the following calculations, local values of irradiance determined using Eq. (24) will be denoted $G^{h}(z)$.

Because the radiation field is modified, it is interesting to recalculate in the Lagrangian approach the light absorbed by flowing cells with the irradiance field determined using Eq. (24) instead of Eq. (4). 
To compare with the spatial integration of the LVREA (Eq. (16)), the calculation is expressed in terms of temporal averaging (the new value is noted $\overline{A_{\infty}^{h}}$ ) and is defined as follows:

$\overline{A_{\infty}^{h}}=\lim _{T \rightarrow \infty}\left(E_{a} X \overline{G^{h}}(T)\right)=E_{a} X \overline{G_{\infty}^{h}}$

The previous approach where the radiation field was supposed independent of the flow (Eq. (4)) was also used to calculate the corresponding MVREA $\left(\overline{A_{\infty}}\right)$, rewriting Eq. (17) as

$\overline{A_{\infty}}=E_{a} X \overline{G_{\infty}}=E_{a} X\left[\lim _{T \rightarrow \infty}(\bar{G}(T))\right]$

All of the calculation methods are compared to the results of the spatial integration of the radiative flux on the reactor surface (Eq. (18)). If the formulation is correct, it must satisfy the energetic balance. Some examples of results are given in Fig. 8.

These results confirm that, when neglecting flow effect on radiative transfer in Lagrangian formulation $\left(\overline{A_{\infty}}\right)$, the amount of light

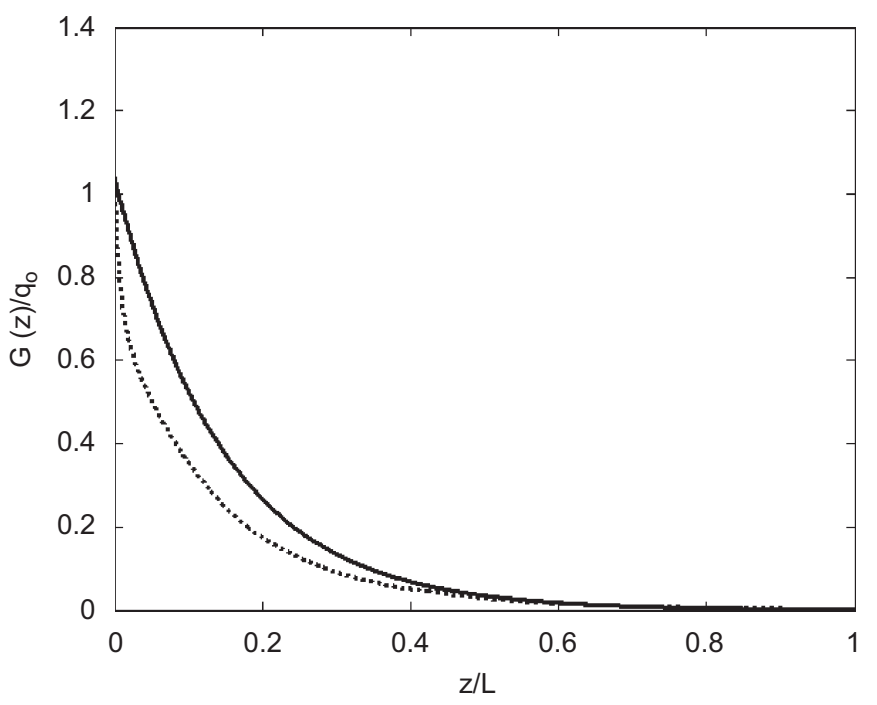

Fig. 7. Example of irradiance field in the torus PBR with $\left(G^{h}(z)\right.$-dotted line) and without $(G(z)$-line) correction due to heterogeneous local absorption rates $\left(N_{\text {imp }}=300 \mathrm{rpm}-X=1 \mathrm{~g} / \mathrm{l}-q_{\cap}=200 \mu \mathrm{E} \mathrm{m}^{-2} \mathrm{~s}^{-1}\right)$.

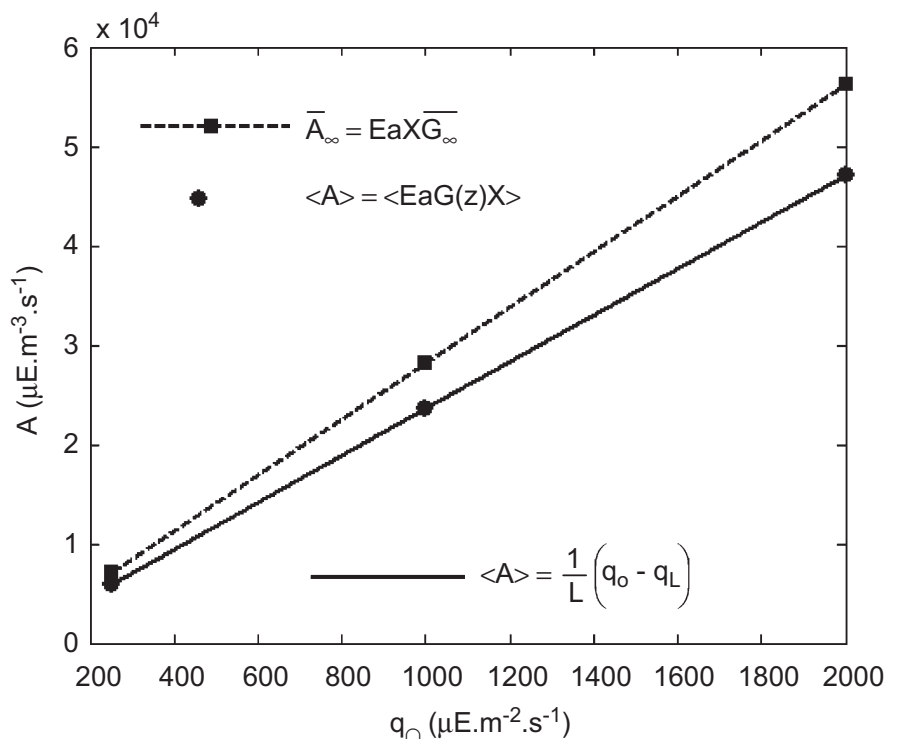

absorbed is still over estimated (the correct value being represented by $\langle A\rangle$, Eq. (18)). Error increases with the light gradient (higher values of $q_{\cap}$ and $X$ ). However, if the $z$-RTD is considered in the radiative transfer resolution $\left(\overline{A_{\infty}^{h}}\right)$, the energetic balance on the photonic phase is verified. To represent the coupling between flow and radiative transfer in PBR, it is therefore necessary to consider the effect of a non-ideal mixing on local absorption coefficient, as by introducing heterogeneous cell distribution along light attenuation direction in radiative transfer resolution. In that case, the Lagrangian formulation is energetically consistent, as it will be with a Eulerian resolution of both radiative transfer and absorbing cell transport. Compared to photocatalytic application, the interest of the method is that it focused only along the depth of culture, where light attenuation occurs in PBRs. Considering the effect of flow on cell concentration only in the light attenuation direction is thus an interesting compromise to integrate flow influence on light availability without a great increase of computation time. This is especially true in the case of one-dimensional attenuation where $h_{T}$ depends only on one coordinate. A Lagrangian formulation can therefore be kept, which is interesting for a further coupling to a dynamical biological model.

In Eq. (21), it was shown that the introduction of $h_{T}(z)$ in the spatial integration has allowed the representation of non-ideal mixing on the mean available irradiance. Flow influence was, this way, represented in the spatial averaging. Because the LVREA is based on both local irradiance and concentration, $h_{T}(z)$ can be simply introduced by combining Eqs. (16) and (23). To differentiate from previous definition where irradiance field was assumed independent of flow conditions (Eq. (16)), the new value based on Eq. (24) is noted $\left\langle A^{h}\right\rangle$ and is given by

$$
\begin{aligned}
\left\langle A^{h}\right\rangle & =\frac{1}{L} \int_{0}^{L} E_{a} G^{h}(z) X(z) \mathrm{d} z=\frac{E_{a}}{L} \int_{0}^{L} G^{h}(z) h_{T}(z) X \mathrm{~d} z \\
& =\frac{a}{L} \int_{0}^{L} h_{T}(z) G^{h}(z) \mathrm{d} z
\end{aligned}
$$

For convenience, this equation can also be expressed as Eq. (16): $\left\langle A^{h}\right\rangle=a\left\langle h_{T} G^{h}\right\rangle=\left\langle E_{a} h_{T} G^{h} X\right\rangle$

As can be seen in Fig. 8, the results of $\left\langle A^{h}\right\rangle$ are exactly the same as those obtained with the temporal averaging using the Lagrangian approach $\left(\overline{A_{\infty}^{h}}\right)$. Again, introduction of $h_{T}(z)$ in the formulation allows thus to reconcile both spatial and temporal integrations on

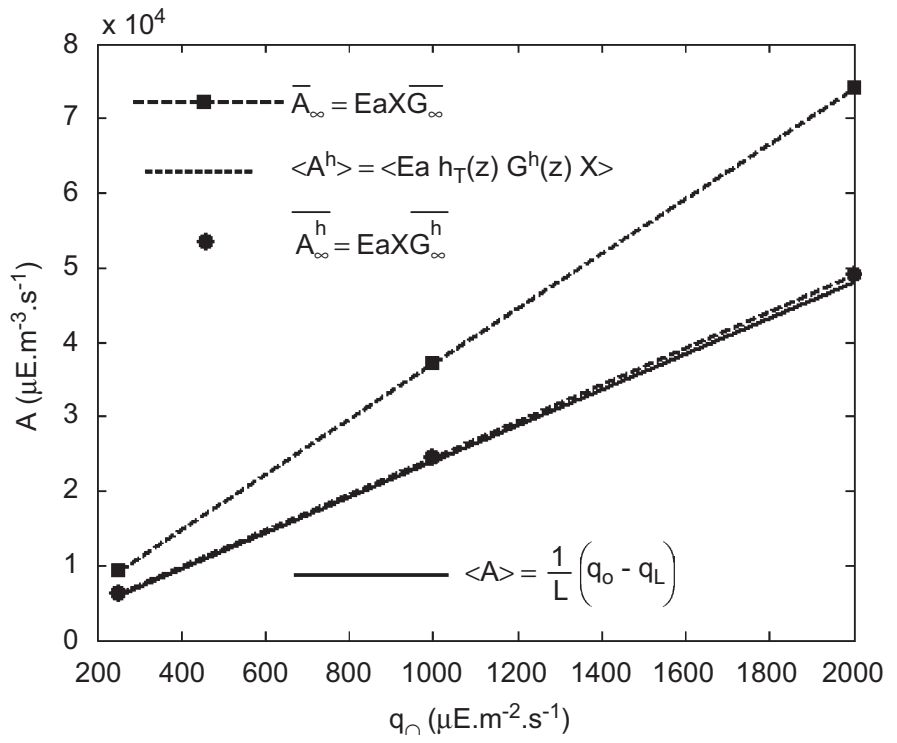

Fig. 8. Results of the different calculations of the average value of $A$ for $X=0.5 \mathrm{~g} / \mathrm{l}$ (left) and $X=1.2 \mathrm{~g} / \mathrm{l}$ (right). 
trajectories, which is interesting in terms of saving calculation time, resolution of Eq. (28) including Eq. (24) to determine $G^{h}(z)$ being almost instantaneous on a standard PC (compared to a few hours for calculating the result of the Lagrangian approach with Eq. (25)).

Even if the use of $h_{T}(z)$ appears relatively simple, its determination in a given geometry implies previous tedious hydrodynamic investigation. Such characterization effort is, however, not necessarily justified. Because both formulations are valid, if the PBR running is only dependent on the light input, representation of flow influence on light transfer would thus have no interest. This point will be discussed in the next section. An accurate representation of the light received can however be relevant, as for example in the investigation of light regime obtained in PBR using a Lagrangian formulation to access cell trajectories.

\section{Growth simulation under light-limited conditions}

\subsection{Growth modeling}

\subsubsection{Overview of the theoretical investigation of flow influence on} photosynthetic growth

In order to investigate the role of hydrodynamics on the photosynthetic conversion in PBR, different modeling approaches have been conducted. All are based on the coupling between the radiative model and a classical photosynthetic growth model, neglecting any possible L/D cycles effects. As a reference, the biomass increase is obtained by a standard mass balance on the reactor, assuming perfectly mixed conditions. To introduce possible effects of a non-ideal mixing, the Lagrangian formulation is next introduced.

\subsubsection{Kinetic model for photosynthetic growth}

To represent light-limited growth, available light must be related by a kinetic model to the resulting photosynthetic growth. Considering previous conclusions, it would be more judicious to use a model based on the LVREA (A), as commonly applied in photoreaction. By definition, such local formulation is adapted to the representation of a heterogeneous rate of radiative energy absorption (Eq. (16)). However, this rigorous approach supposes to formulate the coupling by well defined energetic and quantum yields as proposed for prokaryotic micro-organisms (Cornet et al., 2001, 2003). This requires using sophisticated methods such as metabolic flux analysis or linear thermodynamics of irreversible processes, which are unfortunately not available today for this purpose, considering eukaryotic microorganisms. For simplification, a classical formulation will be retained, based on the local irradiance $\mathrm{G}$. It enables to take into account the decrease in energetic yield of conversion when increasing the LVREA (or the irradiance in this case) from a kinetic model of representation, so leading to distinguish the part of wasted photons as heat or fluorescence.

The growth model represents the photosynthetic conversion of Chlamydomonas reinhardtii $137 \mathrm{c}$, in terms of specific growth rate $\mu$ as a function of irradiance. Experimental growth rates were measured in the torus PBR, because of the high control of the irradiance field provided by such a geometry (Pottier et al., 2005). A complete description of the procedure is given in Appendix 2. Results are presented in Fig. 9. Values of the specific growth rate were fitted using a kinetic model with inhibitory term (Aiba, 1982) to characterize the small decrease of growth rate observed for irradiance higher than $400 \mu \mathrm{E} \mathrm{m}^{-2} \mathrm{~s}^{-1}$. The corresponding equation is

$\mu(G)=\mu_{m} \frac{G}{K_{\mathrm{I}}+G+\frac{G^{2}}{K_{\mathrm{II}}}}-\mu_{S}$

For $C$. reinhardtii, values of the model are $K_{\mathrm{I}}=69.75 \mu \mathrm{E} \mathrm{m}^{-2} \mathrm{~s}^{-1}$, $K_{\mathrm{II}}=2509.66 \mu \mathrm{E} \mathrm{m}^{-2} \mathrm{~s}^{-1}, \mu_{m}=0.2479 \mathrm{~h}^{-1}, \mu_{s}=0.0531 \mathrm{~h}^{-1}$. This gives

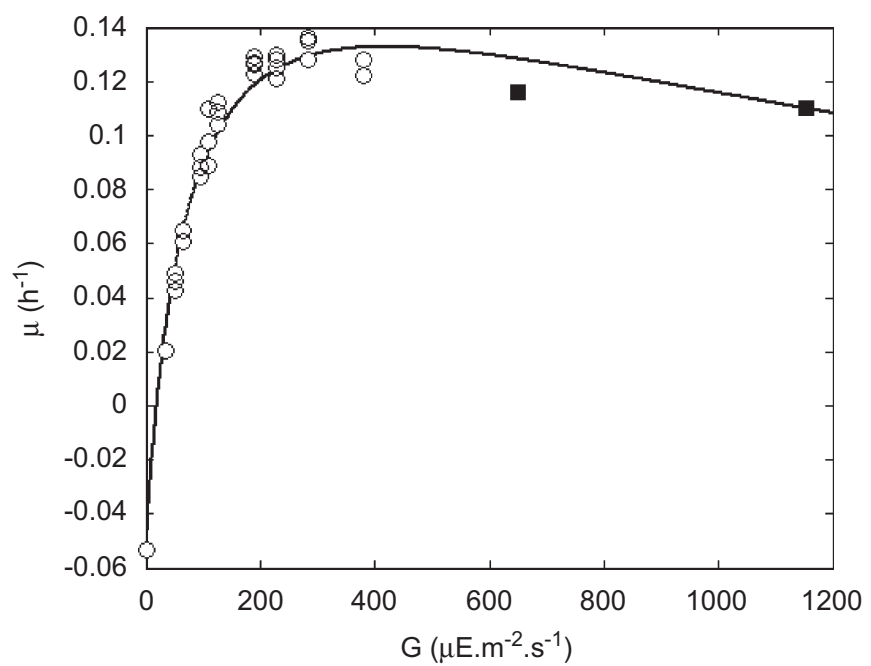

Fig. 9. Photosynthetic growth rate of Chlamydomonas reinhardtii (white circles: experimental results obtained in the torus PBR-black squares: additional data obtained from Janssen et al., 2000-curve: kinetic model fitting).

a maximum growth rate of $0.133 \mathrm{~h}^{-1}$ at $400 \mu \mathrm{E} \mathrm{m}^{-2} \mathrm{~s}^{-1}$. This characteristic value will be noted $\mu_{\text {sat }}$ and used later to adimensionalize results.

\subsection{Growth simulation results}

Association of the radiative model (Eq. (4)) with the photosynthetic growth model (Eq. (29)) allows to simulate light-limited cultivation, by solving the mass-balance equation on the reactor:

$\frac{\mathrm{d} X}{\mathrm{~d} t}=\mu_{a} X-D X$

where $\mu_{a}$ is the averaged growth rate.

Calculation of an averaged growth rate is necessary because of the local nature of the photosynthetic response due to irradiance attenuation. This is usually obtained by a spatial averaging of local growth rates that gives for one-dimensional attenuation (Cornet et al., 1998; Yun and Park, 2003):

$\mu_{a}=\langle\mu\rangle=\frac{1}{L} \int_{L} \mu(G(z)) \mathrm{d} z$

An example of results obtained in the case of the torus PBR with Chlamydomonas reinhardtii is given in Fig. 10(a). This is a well known result, where light limitation effect is clearly shown, with a maximum biomass concentration completely dependent on the light input. The photosynthetic conversion being limited, interest of increasing the radiative flux density decreases progressively. As shown in Fig. 10(b), because high irradiance results in high biomass concentration, strong light gradients are achieved. For the maximum of radiative flux investigated, only a small part of the reactor remains illuminated. Local photosynthetic growth rates corresponding to some examples of maximum concentrations are represented in Fig. 10(c). This repartition can be used to divide reactor depth in two zones: one where photosynthetic activity is higher than respiration (resulting in $\mu>0)$, and one where respiration is higher $(\mu<0)$. Because of the definition of the growth rate averaging (Eq. (31)), those two zones compensate each other when a maximum concentration is obtained. The separation between those two zones defines the dark and illuminated regions, the residence time in each defining periods of $\mathrm{L} / \mathrm{D}$ cycles. If the case of high radiative flux density is considered (case $\mathrm{n}^{\circ} 3$ in Fig. 10), a heterogeneous local response is observed, 
a

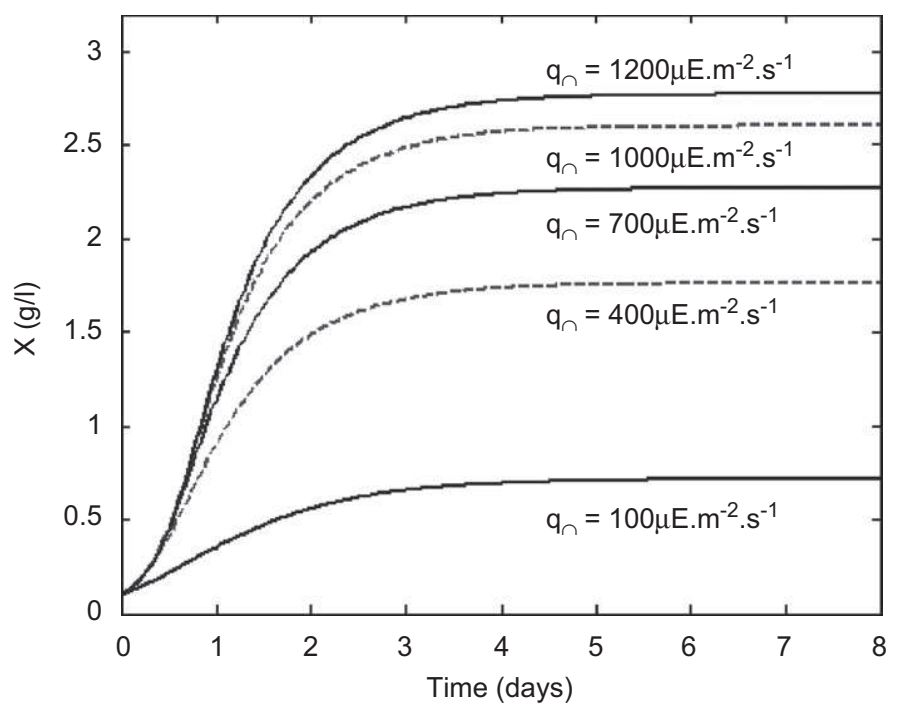

C

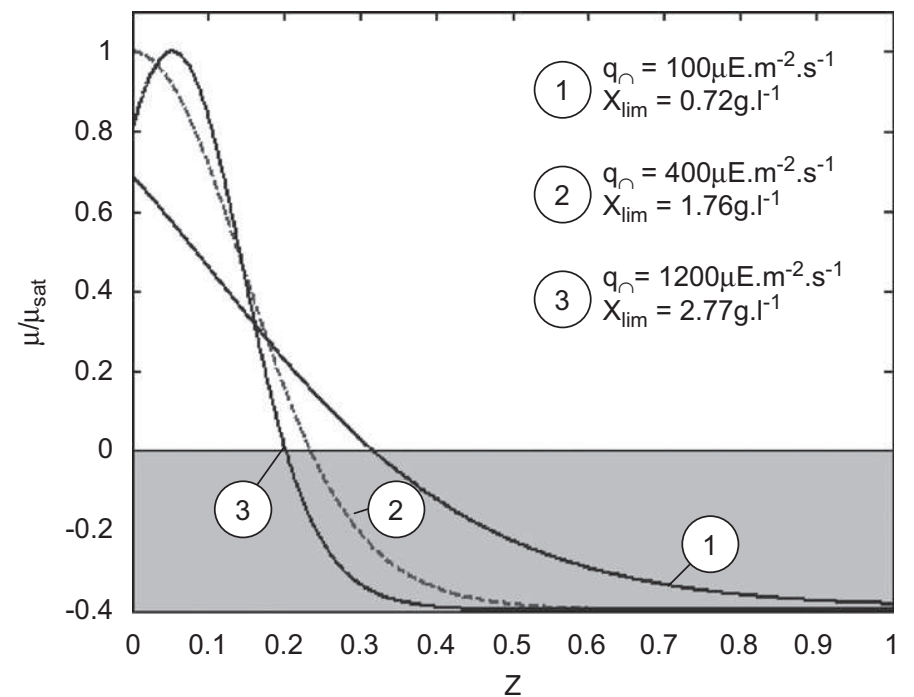

b

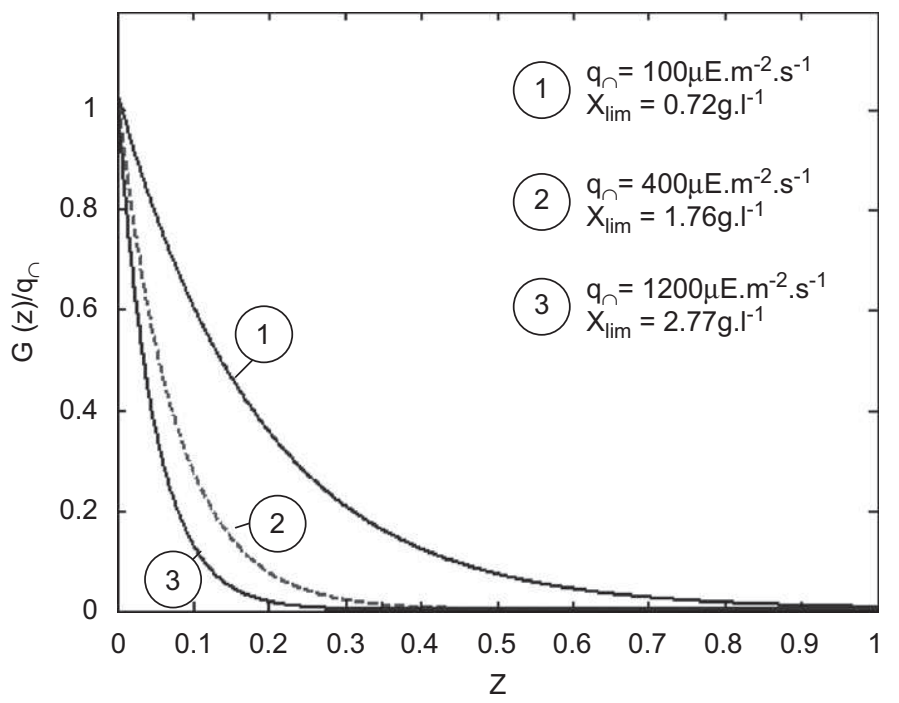

d

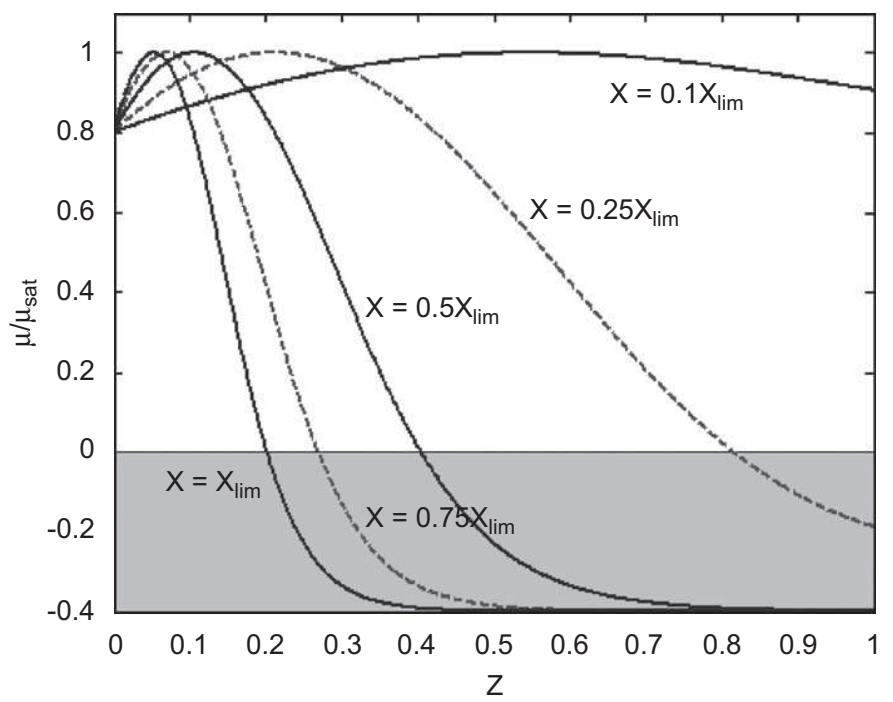

Fig. 10. Simulation of light-limited growth of Chlamydomonas reinhardtii in the torus PBR operated in batch mode, without flow influence ((a) biomass concentration evolution; (b) example of irradiance fields for given absorbing conditions; (c) example of local growth rate repartition for given absorbing conditions; (d) example of evolution of the local growth rate during a batch culture for $q_{\cap}=1200 \mu \mathrm{E} \mathrm{m}^{-2} \mathrm{~s}^{-1}$ ).

with over-saturating irradiance near the optical surface. Because of the complex role of the time of exposure on the photoinhibition process, influence of this metabolic zone remains rather misunderstood. In addition, because of the progressive increase of concentration in batch conditions, those metabolic zones will dynamically change, as shown in Fig. 10(d). Considering the culture duration which is only of few days for $C$. reinhardtii, such results illustrate the great difference of radiative conditions that photosynthetic cells can encounter along a batch cultivation in PBR, as compared to natural conditions. Even if illumination conditions in nature are also not permanent, dynamics of evolution are in the general case obviously slower (change of seasons, diurnal cycle). Conditions in PBRs can thus be regarded as an extreme case of the photosynthetic growth, with faster evolutions and very specific light regimes. This confirms interest of fundamental studies focused on the specific behavior that photosynthetic cells can exhibit when cultivated in PBR.

In a second step, the photosynthetic growth model (Eq. (29)) has been associated to the Lagrangian determination of cell trajectories.
Because of preceding remarks, the radiation field is solved using Eq. (24) with corresponding $h_{T}(z)$ distribution so as to obtain an accurate formulation of the Lagrangian approach. This will allow to investigate the possible influence of a heterogeneous light access on PBR efficiency (global effect).

Because the Lagrangian approach gives cell displacement along the light gradient, light history $G(t)$ is known. Using the growth model, instantaneous responses characterized by $\mu(G(t))$ are determined using Eq. (29). The increase in biomass can then be calculated step by step, according to the following expression:

$$
\begin{aligned}
X(t+\Delta t) & =X(t)+[\mu(t)-D] X(t) \Delta t \\
& =X(t)[1+(\mu(G(t))-D) \Delta t]
\end{aligned}
$$

where $\Delta T$ represents the time between two successive positions as used in the Lagrangian simulation (Eq. (5)).

This approach was already applied in another PBR geometry (Pruvost et al., 2002a). The main drawback is in the very long computation time necessary to simulate a culture cultivation. Time step 


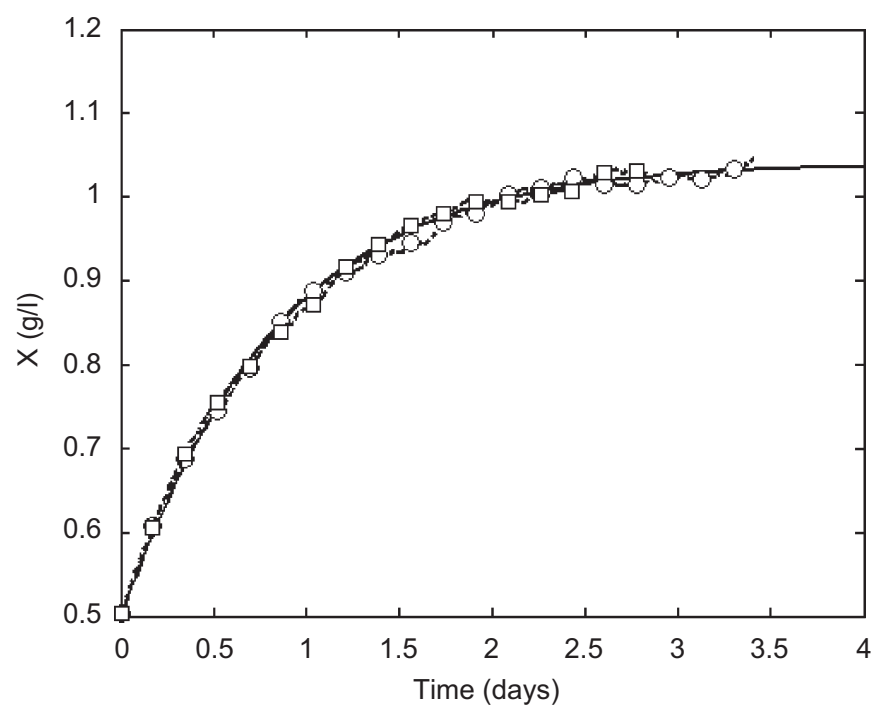

Fig. 11. Simulation of light-limited growth of Chlamydomonas reinhardtii in the torus PBR operated in batch mode $\left(N=300 \mathrm{rpm}-q_{\cap}=300 \mu \mathrm{E} \mathrm{m}^{-2} \mathrm{~s}^{-1}\right)$, with flow influence (square: cell no. 1 with Lagrangian simulation; circle: cell no. 2 with Lagrangian simulation; line: spatial resolution with introduction of $h_{T}(z)$ ).

in the Lagrangian calculation is indeed of the order of the hundredth of second to have an accurate representation of trajectories, while the growth to simulate is over a period of several days. In addition, because of the coupling between radiative transfer and flow conditions, the irradiance field is obtained by a numerical resolution of the set of differential equations governing the two-flux model (Eq. (24)), which has to be repeated for each evolution of biomass concentration. A typical simulation requests several days of computing (on a Pentium PC, $3.2 \mathrm{GHz}$ ). With this kind of formulation, process optimization by testing various conditions is unrealistic.

The intrinsic advantage is in the possibility of simulating individual cells. An example is given for two cells with different trajectories (Fig. 11). It can be noticed that those two examples give the same limiting concentration, with an almost identical growth, despite their difference in history. This is easily explained by the difference in timescales between light access, which is very short, compared to the long duration of the cultivation. Even if each cell has a different history, a similar evolution is observed on the timescale of the total growth.

To reduce computation time, it appears interesting to introduce $h_{T}(z)$ in the calculation of the mean growth rate, as applied for the light availability (Eq. (21)). Local growth rates can thus be weighted by the corresponding value of $h_{T}(z)$, which gives the following expression:

$\mu_{a}=\frac{1}{L} \int_{L} h_{T}(z) \mu\left(G^{h}(z)\right) \mathrm{d} z$

Using this expression, a modeling approach similar to the one conducted at the beginning of this section (without flow influence) can be proposed, based on spatial averaging with consideration of flow influence. Because the mean growth rate is known, biomass concentration can be simply obtained by solving Eq. (30), without the request of a Lagrangian step-by-step resolution (Eq. (32)). In that simulation, Eq. (33) is substituted to Eq. (31) for the calculation of the mean growth rate, and Eq. (24) is used instead of Eq. (4) for the radiative transfer to consider mixing influence. The growth model remains unchanged and is given by Eq. (29). Result obtained for the same conditions as the previous Lagrangian simulation is added in Fig. 11. A concentration evolution similar to the one with the Lagrangian formulation is achieved. As for the calculation of the mean amount of light absorbed by flowing cells, introduction of $h_{T}(z)$ en-

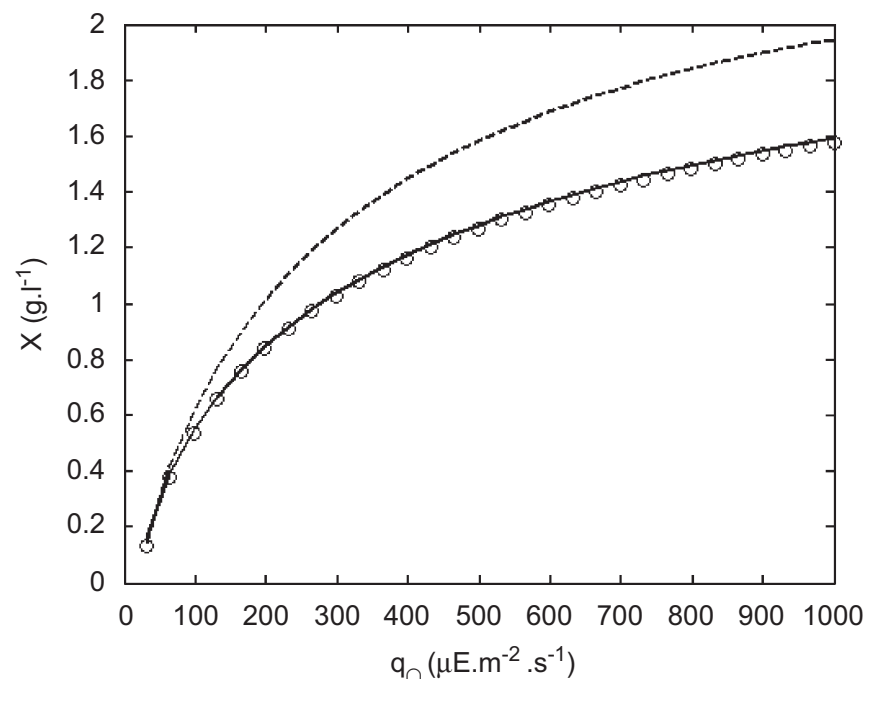

Fig. 12. Maximum biomass concentrations obtained in the torus PBR in batch mode as a function of radiative flux (line: simulation without flow influence; circles: simulation with the Lagrangian approach with consideration of mixing influence on radiative transfer; dotted line: simulation with the Lagrangian approach without consideration of mixing influence on radiative transfer).

ables to use a classic (spatial) resolution, instead of the Lagrangian formulation (Eq. (32)), while taking light access influence into account. Because in that case, the problem boils down to a classical set of differential equations that can be solved with usual numerical resolution procedure (ode113 function in Matlab ${ }^{\circledR}$ software), this greatly reduces computing time (a few seconds).

This procedure has been retained to investigate influence of radiative flux on photosynthetic growth in the torus PBR operating in batch mode. Only biomass concentrations achieved at the plateau (full limitation by light) are presented. A comparison is made with simulations where flow influence on the light transfer is neglected. This is simply obtained by using the analytical solution of the irradiance field when neglecting flow influence (Eq. (4)) instead of solving the differential form of the two-flux model (Eq. (24)). Photosynthetic growth remains unchanged and is given by Eq. (33) with corresponding value of $h_{T}(z)$. To give an additional illustration, predictions assuming perfectly mixed conditions are also given. No flow influence is thus introduced, light transfer being determined by Eq. (4), and the growth by Eq. (29). Comparison between these three different formulations of growth modeling is given in Fig. 12. As already observed in the section devoted to the investigation of the light availability, neglecting flow effect results in an increase in the final concentration achieved, which would be interpreted as a possible global effect of the flow on reactor efficiency. This can be seen in Fig. 12, where the increase in concentration is significant, especially for high PFDs. However, as already concluded, those results are not correct, due to the methodic error that appears when disconnecting radiative transfer from flow influence on local absorption. It must be noticed that those results partly explain the great difference observed between two flows in a preceding study by the authors (Pruvost et al., 2002a).

If the flow effect on light transfer is accurately represented in the Lagrangian formulation, no influence of hydrodynamics can occur, exactly the same results being achieved for the photosynthetic growth. This is an important conclusion in the purpose of modifying mixing conditions to optimize light conversion in PBR. Indeed, as can be seen in Eq. (33), if the residence time is heterogeneous, one would expect to increase the average growth rate in the reactor, by enhancing, thanks to mixing, residence time where the local growth rate was higher (by finding a favorable $h_{T}(z)$ distribution in the $\mu(z)$ weighing). Because the growth rate in PBR is always 
heterogeneous, such a solution could be applied in all cases. However, as results allow to conclude, weighing of the local growth rate with the heterogeneous residence time is perfectly counterbalanced by the modification of local value of absorption rates, resulting in a different radiative transfer inside the reactor, which in turn affects local growth rates. This conclusion is obvious if the energetic of the system is considered, the light absorbed being only fixed by the radiative energy input (Appendix A). If the energetic yield is not modified by dynamic kinetic coupling, growth rate is only fixed by the light available. Modification of hydrodynamic conditions in order to generate a heterogeneous residence time along the depth of culture so as to promote high values of local photosynthetic conversion rates (the "global effect") is then useless.

\section{Conclusion}

The coupling of flow conditions with light transfer in PBR has been investigated, using a fully theoretical approach integrating a radiative model with a Lagrangian modeling of cell trajectories inside the reactor.

Two different ways of calculation of the mean amount of light absorbed in the culture were compared. The first is based on a spatial integration of the radiation field, and the second considers flow effect, by calculating the light absorbed with respect to time by cells flowing in the reactor. Energetic balances on both material and photonic phases assume values to be equal. Results have shown that a "simple" (but usually employed in PBR application) linear association was not sufficient. It was necessary to formulate all the effects of a non-ideal mixing, including a modification of the local volumetric rate of absorption due to heterogeneity in the residence time of cells in given depths of culture. Neglecting this effect leads to an energetic imbalance (i.e., a violation of the first principle of thermodynamics), resulting in a wrong representation of light availability in the reactor.

Even if the transport of the absorbing phase can be solved in a Lagrangian approach by considering thousands of individual cells, its coupling with radiative transfer is unrealistic for PBR application, especially if a growth model has to be introduced for PBR running modeling. Considering however the particular case of one-dimensional hypothesis, a simplified solution was proposed. It is based on a correction of radiative transfer with respect to the time spent by flowing cells along the depth of culture. Cell trajectories determination allows to determine a residence time distribution along light attenuation, the introduction of which in the radiative model leads to an energetically valid formulation of the Lagrangian characterization of light regimes encountered by flowing cells in the PBR.

The Lagrangian method is next extended to simulate photosynthetic growth in a torus PBR. Using a photosynthetic growth of the ideal type (without dynamic effect of L/D cycles), results show that PBR efficiency is only a function of the light input, and that mixing has no effect on global light conversion in the PBR. For a given photosynthetic microorganism species, the PBR running is only imposed by the energetic balance on the photonic phase.

The only effect that can be expected from hydrodynamics coupling in PBR light use is in the alteration of the photosynthetic conversion due to the $\mathrm{L} / \mathrm{D}$ cycles effect. If a dynamic kinetic coupling exists between biological response and fluctuating light regimes encountered by flowing cells, PBR efficiency will be modified for given illumination conditions, a non- linearity being added in light conversion (the sole one, no primary coupling on mean light availability being possible). Dynamic kinetic coupling in PBRs remains however to be clarified because of its complexity, a deep characterization being indeed necessary to elucidate relevant dynamic mechanisms of photosynthetic apparatus when submitted to specific fluctuating light regimes obtained in PBRs. For physical aspects (L/D cycles char- acterization), the Lagrangian approach, with a rigorous treatment of the radiative transfer problem, is well adapted. Because it allows to represent cell history, a further coupling with kinetic models of photosynthesis is direct, opening perspectives to adapt light regimes in PBRs with respect to biological response timescales.

\section{Notation}

a volumetric absorption coefficient, $\mathrm{m}^{-1}$

$a_{S} \quad$ specific illuminated area, $\mathrm{m}^{-1}$

A local volumetric rate of radiant energy absorbed (LVREA), $\mathrm{Wm}^{-3}$

$b$ backscattering fraction for two-flux method, dimensionless

$D \quad$ dilution rate, $\mathrm{s}^{-1}$

e Gaussian distributed random function, $\mathrm{m} \mathrm{s}^{-1}$

$\dot{e}_{U} \quad$ mass internal energy density, $\mathrm{J} \mathrm{kg}^{-1}$

$E_{a} \quad$ mass absorption coefficient, $\mathrm{m}^{2} \mathrm{~kg}^{-1}$

$E_{S} \quad$ mass scattering coefficient, $\mathrm{m}^{2} \mathrm{~kg}^{-1}$

$E_{\mathrm{TOT}} \quad$ volumetric total energy, $\mathrm{J} \mathrm{m}^{-3}$

$f \quad$ weight linked to spatial correlation in fluctuating velocity determination, dimensionless (Eq. (7))

$g \quad$ weight linked to the random part of the fluctuating velocity, dimensionless (Eq. (7))

G irradiance, $\mathrm{W} \mathrm{m}^{-2}$

$G_{\lambda} \quad$ spectral irradiance, $\mathrm{W} \mathrm{m}^{-3}$

$h_{T} \quad$ relative residence time along the depth of culture, dimensionless (Eq. (20))

I specific intensity (radiance), $\mathrm{W} \mathrm{m}^{-2}$

$I_{\lambda} \quad$ spectral specific intensity (radiance), $\mathrm{W} \mathrm{m}^{-3}$

$J_{\text {ETOT }} \quad$ total energy flux density, $\mathrm{W} \mathrm{m}^{-2}$

$k$ turbulent kinetic energy, $\mathrm{m}^{2} \mathrm{~s}^{-2}$

$L \quad$ photobioreactor depth, $\mathrm{m}$

$L_{e} \quad$ integral length scale of turbulence, $\mathrm{m}$

$N \quad$ number of cells considered in ensemble averaging (Eq. (11))

$N_{\text {imp }} \quad$ impeller rotation speed, rpm

$P \quad$ position of a elementary fluid element (Eq. (5))

$q \quad$ radiant energy flux density, $\mathrm{Wm}^{-2}$

$q_{\cap} \quad$ incident hemispherical radiant energy flux density, $\mathrm{W} \mathrm{m}^{-2}$

$\hat{Q} \quad$ volumetric heat power, $\mathrm{W} \mathrm{m}^{-3}$

$r \quad$ distance traveled from an initial position in the Lagrangian calculation, $\mathrm{m}$

$r_{X} \quad$ volumetric biomass growth rate, $\mathrm{kg} \mathrm{m}^{-3} \mathrm{~s}^{-1}$

$R e=U_{0} L / v \quad$ Reynolds number, dimensionless

$S$ surface, $\mathrm{m}^{2}$

$S_{0} \quad$ PBR illuminated surface, $\mathrm{m}^{2}$

$u^{\prime} \quad$ fluctuating fluid velocity, $\mathrm{m} \mathrm{s}^{-1}$ (Eq. (6))

$U \quad$ instantaneous fluid velocity, $\mathrm{m} \mathrm{s}^{-1}$

$U_{0} \quad$ mean bulk velocity, $\mathrm{m} \mathrm{s}^{-1}$

$t \quad$ time, $s$

$V \quad$ volume, $\mathrm{m}^{3}$

$w \quad$ Maxwell electromagnetic energy density, $\mathrm{J} \mathrm{m}^{-3}$

$\hat{W} \quad$ volumetric mechanic power input, $\mathrm{Wm}^{-3}$

$X \quad$ biomass concentration, $\mathrm{kg} \mathrm{m}^{-3}$

$z \quad$ distance along light gradient direction, $\mathrm{m}$

Greek symbols

$\alpha \quad$ linear scattering modulus, dimensionless

$\delta \quad$ two-flux extinction coefficient, $\mathrm{m}^{-1}$

$\Delta t \quad$ time step in the Lagrangian calculation, s (Eq. (5)) 


\section{Subscripts}

$\infty$

h

denotes value obtained after an infinite period of circulation (Eq. (14)) denotes results obtained by weighting local values of irradiance in the integration by $h_{T}(z)$ (Eq. (21))

\section{Superscripts}

$+\quad$ forward direction for radiative transfer (see Eq. (1))

- $\quad$ backward direction for radiative transfer (see Eq. (1))

$h \quad$ denotes results obtained using Eq. (24) for radiative transfer resolution

Other symbol

$$
\begin{array}{ll}
\langle\rangle & \text { spatial averaging } \\
- & \text { Time averaging }
\end{array}
$$

Note: radiation properties must be defined in SI units. Because photosynthesis is rather sensitive to photon flux density, the mole of photons is commonly preferred for kinetic purposes in PBR applications. The conversion factor for conventional energy unit, considering the wavelength $\lambda$. is 1 mole of photon $=1$ Einstein $(E)=(0.1196 / \lambda) \mathrm{J}$.

\section{Appendix A. Local and spatial-averaged energetic balances for photobioreactors}

\section{A.1. Local balances}

As it is well known from classical textbooks (Bejan, 1996; Bird et al., 2002; De Groot and Mazur, 1984), it is not formally possible to obtain a complete understanding of any process without an energetic treatment, particularly, in properly defining the so-called internal energy and total energy balances. Photobioreactors belong to a class of problem in which a reactive material phase interacts with an electromagnetic (or a photonic) phase. This weak coupling was investigated by the author (Cornet, 2005), clearly establishing the partition of the total energy balance from a comprehensive and self-consistent definition of the internal energy balance for the material phase. In the classical Eulerian formalism, neglecting the thermal radiant emission at room temperature, the total energy balance for the material phase is straightforward:

$\frac{\partial}{\partial t}\left[\frac{1}{2} \rho_{f} U^{2}+\rho_{f} \dot{e}_{U}+\rho_{f} \dot{\phi}\right]=-\nabla \cdot \mathbf{J}_{\mathrm{E}_{\mathrm{TOT}}}+A$

in which the complete definition of the flux density $\mathbf{J}_{\mathrm{ETOT}}$ can be found in the paper of Cornet (2005).

Likewise for the photonic phase:

$\frac{\partial w}{\partial t}=-\nabla \cdot \mathbf{q}-A$ in which $A$ is the so-called LVREA and q the radiative flux density or PFD, characterizing the radiation field from:

$\mathbf{q}=\iint_{\Omega} \int_{\lambda} I_{\lambda} \cos \Theta \mathrm{d} \Omega \mathrm{d} \lambda$

$G_{\lambda}=\iint_{\Omega} I_{\lambda} \mathrm{d} \Omega$

$A=\int_{\lambda} a_{\lambda} G_{\lambda} \mathrm{d} \lambda$

Clearly, the volumetric absorption coefficient, a ,enables to distinguish between the irradiance $G$ which is the radiant energy available at any point in the PBR (photonic phase) and the actual local volumetric rate of radiant energy absorbed (LVREA) A by the material phase, i.e., the radiant energy used for the photosensitized reactions. It is noteworthy that $a=E_{a} X$ is a radiative property of the material phase; the mass absorption coefficient $E_{a}$ being an intrinsic property of each cell alone (in case of independent scattering), obtained by applying the generalized Lorenz-Mie theory (Pottier et al., 2005). The LVREA should then be used in properly formulating any kinetic coupling or energetic application in PBR (Cassano et al., 1995; Cornet et al., 2001, 2003). It represents indeed a source/sink term in the two balances (A1)-(A2), i.e., the radiant energy which is actually exchanged between the material and the photonic phases. Obviously, the first principle of thermodynamics states that the total energy is conserved, and then, the LVREA, A, vanishes when adding the total energy balances for each phase leading to verify in the PBR that:

$\frac{\partial}{\partial t}\left[\frac{1}{2} \rho_{f} U^{2}+\rho_{f} \dot{e}_{U}+\rho_{f} \dot{\phi}+w\right]=-\nabla \cdot\left[\mathbf{J}_{E_{\mathrm{TOT}}}+\mathbf{q}\right]=\frac{\partial E_{\mathrm{TOT}}}{\partial t}$

as it should be.

\section{A.2. Spatial-averaged balances}

Engineering applications are addressed indeed by the spatial averaging technique giving actual information at the scale of the PBR, namely the mean averaged productivity $\left\langle r_{X}\right\rangle$ and the mean averaged rate of radiant energy absorbed (MVREA) $\langle A\rangle$. This method must be applied easily to Eq. (A1) giving the so-called total energy balance for the material phase of a perfectly mixed PBR in the general form:

$\frac{\partial E_{\mathrm{TOT}}}{\partial t}=\Delta\left[\mathbf{J}_{E_{\mathrm{TOT}}}\right]+\sum \hat{W}+\sum \hat{\mathbf{Q}}+\langle A\rangle$

in which

$\langle A\rangle=\frac{1}{V} \iiint_{V} A \mathrm{~d} V=\frac{1}{V} \iiint_{V} a G \mathrm{~d} V$

It must be noted in Eq. (A.5) that a detailed discussion about the development of the terms $E_{\mathrm{TOT}}$ and $\mathbf{J}_{E_{\mathrm{TOT}}}$, depending of any thermodynamic assumption for the reactor behavior, is available in Bird et al. (2002), but does not appear necessarily in this appendix. In the same way, the total energy balance (A.2) for the photonic phase may be averaged onto the PBR, which gives with a classical pseudosteady-state hypothesis and using the Gauss divergence theorem:

$-\frac{1}{V} \iiint_{V} \nabla \cdot \mathbf{q} \mathrm{d} V=\langle A\rangle=-\frac{1}{V} \oiint \mathbf{q} \cdot \mathrm{d} \mathbf{S}$

This important result demonstrates that, at the scale of the whole reactor, in order to verify the total energy conservation on both phases (no source or sink term), i.e., the first principle of thermodynamics, the MVREA $\langle A\rangle$ calculated form the properties of the material phase (respectively, the mass absorption coefficient $E_{a}$ and the biomass concentration $X$, Eq. (A.5)) must be rigorously the same as 
the MVREA calculated from the photonic phase (Eq. (A.6)) in just properly balancing the input and output light flux densities at the boundaries, independently of the material phase as:

$\langle A\rangle=\frac{1}{V} \iiint_{V} a G \mathrm{~d} V=-\frac{1}{V} \oiint \mathbf{q} \cdot \mathrm{d} \mathbf{S}$

As a consequence, the MVREA and then the maximum kinetic performances of any PBR are completely determined by the terms involved on the right-hand side of Eq. (A.7), i.e., the specific illuminated area $a_{S}=S / V$ and the mean incident radiant light flux density onto the reactor $q_{0}$. Thus, as explained in this paper in details, if the coupling between the radiant light and the hydrodynamic fields is rigorously formulated (possibly taking into account a non-homogeneous biomass concentration leading to solve a non-linear radiant light transfer problem), the resulting MVREA must have a unique value, independent in any way of the history of the cells in term of Lagrangian trajectories inside the PBR. This leads to conclude that contrary to many recently published results in the literature, the performances of a PBR can neither be increased (nor decreased) by a primary coupling between the light radiation available and the cell trajectories, unless violating the first principle of thermodynamics.

This becomes particularly obvious in dealing with the very interesting example of a one-dimensional illuminated PBR operating in condition of physical limitation by light (all the photons entering the reactor are used for the photosensitized reactions occurring in the liquid phase). This is a very often encountered situation developed in this paper, first because, the optimal design of (artificial) PBR tends to conceive quasi one-dimensional field of radiation, and second, because in all cases for a given design, the light-limited situation always corresponds to the highest kinetic performances.

In case of quasi one-dimensional attenuation of light in a plane geometry (case of the torus PBR, see Fig. 5), Eq. (A.7) may be rewritten to obtain the following expression (same as Eq. (18) in the text):

$\langle A\rangle=\frac{1}{L} \int_{0}^{L} a(z) G(z) \mathrm{d} z=\frac{1}{V}\left[S_{0}\left(q_{0}-q_{L}\right)\right]=\frac{1}{L}\left(q_{0}-q_{L}\right)$

Additionally, if the PBR operates at its maximum performances in light-limited conditions, the light flux density at the output $q_{L}$ is close to zero, and introducing the specific illuminated area $a_{S}$, one obtains (Cornet et al., 1998):

$\langle A\rangle_{\max }=\frac{1}{L} \int_{0}^{L} a(z) G(z) \mathrm{d} z=a_{S} q_{0}=a_{S}\left(q_{0}^{+}-q_{0}^{\mathrm{ref}}\right)=\frac{2 \alpha}{1+\alpha} a_{S} q_{\cap}$

showing, as explained above that the maximum MVREA in the PBR is completely determined by the light flux boundary conditions $\left(q_{0}\right)$, its geometrical design $\left(a_{S}\right)$ and the radiative properties of the cells $(\alpha)$, and then independent in any way of the hydrodynamics of the material phase. Of course, this conclusion is not true if the hydrodynamics is supposed to modify the coupling (as for example the so-called L/D cycle effects) between LVREA (or rigorously calculated local irradiance $G$ ) and local kinetics.

Finally, it is less obvious to demonstrate the invariance of the MVREA in condition of kinetic regime from Eq. (A.8), i.e., when photons are in excess with a finite value of the hemispherical PFD $q_{L}$ at the rear of the PBR. A simple reasoning would consider that, in any given situation, the MVREA only depends on the constant number of absorbing species of the material phase (in relation to the biomass concentration), but never on their spatial distribution or their trajectories history. This relies on the definition of the absorption coefficient in term of photon capture probability from the transport theory (Preisendorfer, 1957). Nevertheless, a more rigorous approach to this problem is needed, examining a general situation for the coupling between non-ideal mixing with Lagrangian trajectories consid- eration and non-linear radiative transfer field, as examined in this paper.

\section{Appendix B. Experimental characterization of the photosynthetic} growth rate of Chlamydomonas reinhardtii

\section{B.1. Overview of the method using the torus PBR operated in turbidostat mode}

To obtain an accurate measurement of the specific growth rate, cultures were conducted in continuous mode. In addition to obtain a stable biological response, the averaged specific growth rate in the reactor is then equal at equilibrium to the dilution rate that can be controlled with a high accuracy. For PBR, the "substrate" is light, and because of its attenuation, the light available in the culture is different from the incident light flux (an usual confusion when studying photosynthetic microorganisms growth). A correction must thus be applied, based on the radiative transfer model. Values of the radiative parameters presented in Section 2.1 were used for this correction. When stable conditions are calculated, the biomass concentration is measured. The available light in the culture is then obtained by integrating the corresponding attenuation profile (for example $\langle G\rangle / q_{\cap}=0.6$ for $\left.X=0.15 \mathrm{~g} / \mathrm{l}^{-1}\right)$.

The reactor was operated in turbidostat. The feeding flow rate is then regulated so as to keep constant the biomass concentration inside the reactor, biomass concentration being deduced from a light absorption measurement using a quantum sensor placed in the back side of the reactor. This system allows to maintain a very low biomass concentration inside the reactor $(X<0.1 \mathrm{~g} / \mathrm{l})$. A very accurate measurement is thus obtained. Prediction error of the radiative transfer model is indeed negligible at low light attenuation, as well as effects of mixing on a modification of light transfer in the reactor. Calculation of the attenuation profile can thus be conducted even by Eq. (4) or Eq. (24), both equations giving very close results (data not shown). In addition, it must be noticed that, because of the negligible light attenuation inside the reactor, the culture can be supposed fully illuminated, and the biological response observed is thus without dynamic effect.

\section{B.2. Experimental details}

Chlamydomonas reinhardtii was cultivated in a Sueoka high salt (HS) medium (Harris, 1989). The temperature was regulated at $25^{\circ} \mathrm{C}$. The $\mathrm{pH}$ was maintained at 7.0 by automatic injection of $\mathrm{CO}_{2}$, preventing limitation with respect to the carbon source. To determine the attenuation profile, the algal dry weight $(X)$ was determined by filtration through a pre-dried and pre-weighed glass-fiber filter (Whatman GF/F). The filter was dried at $105^{\circ} \mathrm{C}$, allowed to cool in a dessiccator and then weighed again, giving the value of $X$. Measurements were repeated for different incident light flux, up to a radiative flux of $400 \mu \mathrm{E} \mathrm{m}^{-2} \mathrm{~s}^{-1}$ (Pottier, 2005). Because this corresponds to the saturating light for Chlamydomonas reinhardtii, two values issued from Janssen et al. (2000a) have been added to illustrate possible interaction between photoinhibition and light access in PBR. A specific measurement was also done without illumination, to determine the respiration rate. Because such characterization cannot be achieved in continuous mode $(\mu<0)$, it was estimated by measuring the loss in dry weight occurring under dark conditions during a short period (a few hours) of a previously grown culture.

\section{References}

Aiba, S., 1982. Growth kinetics of photosynthetic microorganisms. Advances in Biochemical Engineering and Biotechnology 23, 85-156.

Bejan, A 1996. Entropy generation minimization. CRC Press.

Bird, R.B., Stewart, W.E., Lightfoot, E.N., 2002. Transport Phenomena. second ed.. Wiley, Inc., New York. 
Camacho, F.R., Camacho, F.G., Sevilla, F.J.M., Chisti, Y., Molina Grima, 2003 A mechanistic model of photosynthesis in microalgae. Biotechnology and Bioengineering 81 (4), 459-473.

Cassano, A.E., Martin, C.A., Brandi, R.J., Alfano, O.M., 1995. Photoreactor analysis and design: fundamentals and applications. Industrial and Engineering Chemistry Research 34 (7), 2155-2201.

Coelho, P.J., 2007. Numerical simulation of the interaction between turbulence and radiation in reactive flows. Progress in Energy and Combustion Science 33 (4), 311-383.

Cornet, J.F., 2005. Theoretical foundations and covariant balances for chemical engineering applications with electromagnetic field. Chemical Engineering Communications 192, 647-666.

Cornet, J.F., Dussap, C.G., Gros, J.B., 1995. A simplified monodimensional approach for modeling coupling between radiant light transfer and growth kinetics in photobioreactors. Chemical Engineering Science 50 (9), 1489-1500.

Cornet, J.F., Dussap, C.G., Gros, J.B., 1998. Kinetics and energetics of photosynthetic micro-organisms in photobioreactors: application to Spirulina growth. Advances in Biochemical Engineering and Biotechnology 59, 155-224.

Cornet, J.F., Dussap, C.G., Leclercq, J.J., 2001. Simulation design, and model based predictive control of photobioreactors. In: Hofman, M., Thonart, P. (Eds.), Focus on biotechnology, Engineering and manufacturing for biotechnology, vol. 4. Kluwer Academic Publishers, Dordrecht, pp. 227-238.

Cornet, J.F., Favier, L., Dussap, C.G., 2003. Modeling stability of photoheterotrophic continuous cultures in photobioreactors. Biotechnology Progress 19 (4) 1216-1227.

De Groot, S.R., Mazur, P., 1984. Non-equilibrium Thermodynamics. second ed. Dover, New York.

Eilers, P.H.C., Peeters, J.C.H., 1993. Dynamic behaviour of a model for photosynthesis and photoinhibition. Ecological Modelling 69, 113-133.

Elyasi, S., Taghipour, F., 2006. Simulation of UV photoreactor for water disinfection in Eulerian framework. Chemical Engineering Science 61 (14), 4741-4749.

Fox, R.O., 2003. In: Press, U. (Ed.), Computational Models for Turbulent Reacting Flows. Cambridge.

Harris, E., 1989. The Chlamydomonas Sourcebook: A Comprehensive Guide To Biology And Laboratory Use. Academic Press, Inc., New York.

Hinze, J.O., 1975. Turbulence. McGraw-Hill, New York.

Janssen, M., De Bresser, L., Baijens, B., Tramper, J., Mur, L.R., Snel, J., Wijffels, R.H. 2000a. Scale-up aspects of photobioreactors: effects of mixing-induced light/dark cycles. Journal of Applied Phycology 12, 225-237.

Janssen, M., Janssen, M.G.J., De Winter, M., Tramper, J., Mur, L.R., Snel, J., Wijffels, R.H., 2000b. Efficiency of light utilization of Chlamydomonas reinhardtii unde medium-duration light/dark cycles. Journal of Biotechnology 78, 123-137.

Janssen, M., Tramper, J., Mur, L.R., Wijffels, R.H., 2003. Enclosed outdoor photobioreactors: Light regime, photosynthetic efficiency, scale-up, and future prospects. Biotechnology and Bioengineering 81 (2), 193-210.

Kondo, T., Wakayama, T., Miyake, J., 2006. Efficient hydrogen production using a multi-layered photobioreactor and a photosynthetic bacterium mutant with reduced pigment. International Journal of Hydrogen Energy 31 (11), 1522-1526.

Lapin, A., Schmid, J., Reuss, M., 2006. Modeling the dynamics of E. coli populations in the three-dimensional turbulent field of a stirred-tank bioreactor-A structured-segregated approach. Chemical Engineering Science 61 (14) 4783-4797.

Larsson, G., Törnkvist, M., Stahl Wernersson, E., Trägardh, C., Noorman, H., Enfors, S.O., 1996. Substrate gradients in bioreactors: origin and consequences. Bioprocess Engineering 14, 281-289.

Luo, H.P., Al-Dahhan, M.H., 2004. Analyzing and modeling of photobioreactors by combining first principles of physiology and hydrodynamics. Biotechnology and Bioengineering 85 (4), 382-393.

Fernandez Sevilla, Luo, H.P., Kemoun, A. Al-Dahhan, M.H., 2003. Analysis of photobioreactors for culturing high-value microalgae and cyanobacteria via an advanced diagnostic technique: CARPT. Chemical Engineering Science 58 (12), 2519-2527.

Merchuk, J.C., Ronen, M., Giris, S., Arad, S., 1998. Light/dark cycles in the growth of the red microalga Porphyridium sp. Biotechnology and Bioengineering 59 (6), 705-713.
Muller-Feuga, A., Le Guedes, R., Pruvost, J., 2003a. Benefits and limitations of modeling for optimization of Porphyridium cruentum cultures in an annular photobioreactor. Journal of Biotechnology 103 (2), 153-163.

Muller-Feuga, A., Pruvost, J., Le Guedes, R., Le Dean, L., Legentilhomme, P., Legrand J., 2003b. Swirling flow implementation in a photobioreactor for batch and continuous cultures of Porphyridium cruentum. Biotechnology and Bioengineering 84 (5), 544-551.

Pahl-Wostl, C., 1992. Dynamic versus static models for photosynthetis. Hydrobiologia 238, 189-196.

Pareek, V.K., Cox, S.J., Brungs, M.P., Young, B., Adesina, A.A., 2003. Computational fluid dynamic (CFD) simulation of a pilot-scale annular bubble column photocatalytic reactor. Chemical Engineering Science 58 (3-6), 859-865.

Perner-Nochta, I., Posten, C., 2007. Simulations of light intensity variation in photobioreactors. Journal of Biotechnology 131 (3), 276-285.

Polle, J.E.W., Kanakagiri, S., Jin, E., Masuda, T., Melis, A., 2002. Truncated chlorophyll antenna size of the photosystems-a practical method to improve microalgal productivity and hydrogen production in mass culture. International Journal of Hydrogen Energy 27 (11-12), 1257-1264.

Pottier, L., 2005. Modélisation de l'effet de l'hydrodynamique sur l'accès à la lumière: application à la croissance photosynthétique dans un réacteur de géométrie torique: Ph.D. Thesis. Nantes University, France.

Pottier, L. Pruvost, J., Deremetz, J. Cornet, J.F. Legrand, J. Dussap, C.G. 2005. A fully predictive model for one-dimensional light attenuation by Chlamydomonas reinhardtii in a torus reactor. Biotechnology and Bioengineering 91 (5), 569-582.

Preisendorfer, R.W., 1957. A mathematical foundation for radiative transfer theory. Journal of Mathematics and Mechanics 6 (6), 685-730.

Pruvost, J., Legrand, J., Legentilhomme, P., Doubliez, L., 2000. Particle Image Velocimetry investigation of the flow-field of a 3D turbulent annular swirling decaying flow induced by means of a tangential inlet. Experiments in Fluids 29 (3), 291-301.

Pruvost, J., Legrand, J., Legentilhomme, P., Muller-Feuga, A., 2002a. Simulation of microalgae growth in limiting light conditions-flow effect. A.I.Ch.E. Journal 48, $1109-1120$.

Pruvost, J., Legrand, J., Legentilhomme, P., Muller-Feuga, A., 2002b. Trajectory Lagrangian model for turbulent swirling flow in an annular cell Comparison with RTD measurements. Chemical Engineering Science 57 (7), 1205-1215.

Pruvost, J., Pottier, L., Legrand, J., 2006. Numerical investigation of hydrodynamic and mixing conditions in a torus photobioreactor. Chemical Engineering Science 61 (14), 4476-4489.

Richmond, A., 2004a. Handbook of Microalgal Culture: Biotechnology And Applied Phycology. Blackwell Sciences Ltd., Oxford.

Richmond, A., 2004b. Principles for attaining maximal microalgal productivity in photobioreactors: an overview. Hydrobiologia 512, 33-37.

Rosello Sastre, R., Csögör, Z., Perner-Nochta, I., Fleck-Schneider, P., Posten, C., 2007. Scale-down of microalgae cultivations in tubular photo-bioreactors-A conceptual approach. Journal of Biotechnology 132 (2), 127-133.

Schmalzriedt, S., Jenne, M., Mauch, K., Reuss, M., 2003. Integration of physiology and fluid dynamics. Advances in Biochemical Engineering and Biotechnology 80 $18-68$.

Spolaore, P., Joannis-Cassan, C., Duran, E., Isambert, A., 2006. Commercial applications of microalgae. Journal of Bioscience and Bioengineering 101 (2), 87-96.

$\mathrm{Wu}$, X., Merchuk, J.C., 2001. A model integrating fluid dynamics in photosynthesis and photoinhibition processes. Chemical Engineering Science 56, 3527-3538.

Wu, X., Merchuk, J.C., 2002. Simulation of algae growth in an bench-scale column reactor. Biotechnology and Bioengineering 80 (2), 156-168.

$\mathrm{Wu}$, X., Merchuk, J.C., 2004. Simulation of algae growth in an bench-scale internal loop airlift reactor. Chemical Engineering Science 59, 2912-2999.

Yoshimoto, N., Sato, T., Kondo, Y., 2005. Dynamic discrete model of flashing light effect in photosynthesis of microalgae. Journal of Applied Phycology 17 (3), 207-214.

Yun, Y.S., Park, J.M., 2003. Kinetic modeling of the light-dependent photosynthetic activity of the green microalga Chlorella vulgaris. Biotechnology and Bioengineering 83 (3), 303-311.

Zonneveld, C., 1998. Photoinhibition as affected by photoacclimation in phytoplankton: a model approach. Journal of Theoretical Biology 193 (1), 115-123. 